\title{
Robust Adaptive Fuzzy Control via State-Dependent Function for Nonlinear Stochastic Large-Scale Systems Subject to Dead Zones
}

\author{
Chang-Qi Zhu and Lei Liu \\ College of Science, Liaoning University of Technology, Jinzhou 121001, Liaoning, China \\ Correspondence should be addressed to Lei Liu; liuleill@live.cn
}

Received 22 May 2021; Accepted 23 September 2021; Published 16 October 2021

Academic Editor: Kalyana C. Veluvolu

Copyright ( 2021 Chang-Qi Zhu and Lei Liu. This is an open access article distributed under the Creative Commons Attribution License, which permits unrestricted use, distribution, and reproduction in any medium, provided the original work is properly cited.

\begin{abstract}
This paper concentrates on the adaptive fuzzy control problem for stochastic nonlinear large-scale systems with constraints and unknown dead zones. By introducing the state-dependent function, the constrained closed-loop system is transformed into a brand-new system without constraints, which can realize the same control objective. Then, fuzzy logic systems (FLSs) are used to identify the unknown nonlinear functions, the dead zone inverse technique is utilized to compensate for the dead zone effect, and a robust adaptive fuzzy control scheme is developed under the backstepping frame. Based on the Lyapunov stability theory, it is proved ultimately that all signals in the closed-loop system are bounded and the tracking errors converge to a small neighborhood of the origin. Finally, an example based on an actual system is given to verify the effectiveness of the proposed control scheme.
\end{abstract}

\section{Introduction}

The nonlinear large-scale systems are a common and significant class of nonlinear systems. As its name implies, it is composed of multiple subsystems, and each subsystem is connected by the terms. Numerous actual systems can be described as nonlinear large-scale systems, such as instance power supply systems, ecological systems, water resources systems, and social-economic systems. The decentralized control study for nonlinear large-scale systems has achieved a lot over the years. In $[1,2]$, the authors studied the adaptive state feedback control problems of nonlinear large-scale systems. Then, the authors in $[3,4]$ have extended the works to the output feedback control problems of nonlinear largescale systems by designing a state observer. To remove the restriction that the system dynamics must be known for the control design exists in [1-4], adaptive fuzzy or neural network (NN) control schemes have been developed [5-12]. In order to achieve the objective in a finite time, the authors in $[5,6,13-15]$ presented the finite-time stabilities for nonlinear large-scale systems with measured and unmeasured states, respectively. To improve the robustness of the system, Tong et al. $[7,8]$ studied the adaptive fuzzy robust control designs for nonlinear large-scale systems with actuator faults and dead zones, respectively. To spare the resources of network communication, the authors in $[9,10]$ studied the event-trigger control problems of nonlinear large-scale systems. However, the results [9-11] will no longer be applicable when stochastic disturbances exist. To deal with this problem, the authors in [16] first presented the method using a quartic Lyapunov function. Based on [16], the authors in $[17,18]$ studied the adaptive fuzzy decentralized control designs for stochastic nonlinear large-scale systems with dynamic uncertainties, the authors in [19] investigated the adaptive fuzzy decentralized control problem for stochastic nonlinear large-scale systems with unknown control directions, and Tong et al. [20] proposed a control algorithm for stochastic nonlinear large-scale systems via the dynamic surface control (DSC) technique.

Note that the above results do not have any requirements for the states of the plants. However, due to the restriction of some physical conditions, the constraints are inevitable in practical engineerings, such as the chemical industry, boiler industry, and robot industry. Therefore, the control study for nonlinear constrained systems becomes necessary, and some meaningful results have been achieved in [21-37]. In 
$[21,22]$, the authors proposed two approaches for uncertain nonlinear systems with output constraints and timevarying output constraints, respectively. To investigate the more complex constrained problem, Liu et al. [23-25] studied adaptive control designs for nonlinear systems with full state constraints. Subsequently, by utilizing the fuzzy logic systems (FLSs) and radial basis function neural networks (RBFNNs) [26, 27], the authors in [30-32] studied adaptive fuzzy or NN control problems of uncertain state-constrained nonlinear systems. Furthermore, the authors in [33] investigated the adaptive fuzzy control design for stochastic high-order nonlinear systems with asymmetric output constraints by designing a novel barrier Lyapunov function (BLF). However, the results above all depend on the feasibility condition, which means that the states of the controlled system cannot be constrained directly. To handle this problem, Zhao et al. $[34,35]$ studied the control problems for single-input and single-output (SISO) nonlinear systems and multiple-input and multipleoutput (MIMO) nonlinear systems with full state constraints by utilizing the nonlinear state-dependent functions, respectively. Then, the authors in [36-38] extended the results in $[34,35]$ to the time-varying state-constrained problems of SISO nonlinear systems and MIMO nonlinear systems, respectively. As another unavoidable factor in the actual system, the dead zone input nonlinearity has been also paid much attention and some valuable results have been achieved in [7, 39-47]. In [39, 40], the authors have investigated the adaptive control problems for nonlinear systems with the symmetric dead zone and asymmetric dead zone, respectively. To deal with the nonlinear dead zone problem, an adaptive $\mathrm{NN}$ control approach has been proposed for uncertain nonlinear systems via the Lagrange mean value theorem in [42]. Furthermore, by using the adaptive estimation mechanism, the dead zone inverse technique has been proposed to compensate the dead zone effect in [7].

It is worth mentioning that most of the results mentioned are for the strict-feedback nonlinear systems instead of nonstrict-feedback nonlinear systems. Different from the strict-feedback nonlinear systems, the unknown nonlinear functions in the nonstrict-feedback nonlinear systems are composed of the whole states. If the traditional control schemes for strict-feedback nonlinear systems are adopted, the algebraic loop problem will be ineluctable. To find a way out of the dilemma, the authors in [46-48] proposed the control algorithms for nonstrictfeedback nonlinear systems via the variable separation technique. Since the variable separation technique requires the nonlinear functions to be strictly monotonic increasing, the authors in $[49,50]$ proposed the novel control algorithms for nonstrict-feedback nonlinear systems by using the property of radial basis function, which do not have any restriction for the nonlinear functions. Motivated by all the mentioned works, an adaptive fuzzy robust control scheme is developed for stochastic state-constrained nonlinear large-scale systems with unknown dead zones. Its main contributions can be summarized as follows.
(1) This paper studied the constrained problem for nonlinear large-scale systems with unknown dead zones. Note that stochastic disturbance is inevitable in engineering practice and the stochastic system is always a research hot spot [51-55]. A novel adaptive law is used to overcome the algebraic loop problem, a variable transformation method is utilized to solve the constrained problem, and a dead zone inverse technique is used to compensate for the dead zone effect.

(2) Although the results in references, like [21-24], are also for the constrained study, they all depend on the feasibility condition, which cannot constrain the states of the system directly, and the developed control scheme in this paper can remove the restriction. On the other hand, the controlled plants in [21-24] are all SISO or MIMO nonlinear systems instead of large-scale nonlinear systems.

\section{Preliminaries}

Consider a class of stochastic nonlinear large-scale systems with unknown dead zones which are composed of $N$ subsystems connected by outputs. The $i$ th subsystem can be expressed as

$$
\sum_{i}\left\{\begin{array}{l}
\mathrm{d} x_{i, 1}=\left[x_{i, 2}+f_{i, 1}\left(x_{i}\right)+\Delta_{i, 1}(\bar{y})\right] \mathrm{d} t+g_{i, 1}\left(x_{i}\right) \mathrm{d} w \\
\mathrm{~d} x_{i, 2}=\left[x_{i, 3}+f_{i, 2}\left(x_{i}\right)+\Delta_{i, 2}(\bar{y})\right] \mathrm{d} t+g_{i, 2}\left(x_{i}\right) \mathrm{d} w \\
\vdots \\
\mathrm{d} x_{i, n_{i}-1}=\left[x_{i, n_{i}}+f_{i, n_{i}-1}\left(x_{i}\right)+\Delta_{i, n_{i}-1}(\bar{y})\right] \mathrm{d} t+g_{i, n_{i}-1}\left(x_{i}\right) \mathrm{d} w \\
\mathrm{~d} x_{i, n_{i}}=\left[D_{i}\left(u_{i}\right)+f_{i, n_{i}}\left(x_{i}\right)+\Delta_{i, n_{i}}(\bar{y})\right] \mathrm{d} t+g_{i, n_{i}}\left(x_{i}\right) \mathrm{d} w \\
y_{i}=x_{i, 1},
\end{array}\right.
$$

where the state vectors of the system are expressed as $x_{i}=\left[x_{i, 1}, x_{i, 2}, \ldots, x_{i, n_{i}}\right]^{T}, \bar{x}_{i, j}=\left[x_{i, 1}, x_{i, 2}, \ldots, x_{i, j}\right]^{T}, 1 \leq i \leq N$ and $1 \leq j \leq n_{i}, u_{i} \in R$ and $y_{i} \in R$ denote the actuator input and sensor output of the system, respectively, $D_{i}\left(u_{i}\right) \in R$ denotes the output of the dead zone, $f_{i, j}\left(x_{i}\right)$ and $g_{i, j}\left(x_{i}\right)$ are the unknown nonlinear functions, $\Delta_{i, j}(\bar{y})$ is the interconnected term which connects each subsystem, and $w$ is an independent $r$-dimensional Wiener process, and we assume that the states of the system can be measured directly.

Remark 1. Different from the research results in references, such as $[1-3,6-8]$, the control study in this paper is to design a robust adaptive fuzzy control method for nonstrictfeedback nonlinear systems. If the traditional control schemes are adopted, the algebraic loop problem will not be able to be avoided. The algebraic loop problem means that if the control design method in strict feedback systems is adopted, the virtual control signal $\alpha_{i}$ in nonstrict-feedback nonlinear systems for the $i$ th subsystem will be a function which contains the entire states $x=\left[x_{1}, x_{2}, \ldots, x_{n}\right]^{T}$, but the states $x_{i+1}, x_{i+2}, \ldots, x_{n}$ are not available at this time. Also, the time-varying state constrained problem is considered in this paper. 
Similar to $[7,40]$, the output of the dead zone is defined as

$$
D_{i}\left(u_{i}\right) \triangleq \begin{cases}m_{i, r}\left(u_{i}-d_{i, r}\right) & \text { if } u_{i} \geq d_{i, r}, \\ 0, & \text { if }-d_{i, l}<u_{i}<d_{i, r} \\ m_{i, l}\left(u_{i}+d_{i, l}\right), & \text { if } u_{i} \leq-d_{i, l} .\end{cases}
$$

In (2), $u_{i}$ is the input to the dead zone, $m_{i, r}$ and $m_{i, l}$ are the slopes of the dead zone, and $d_{i, r}$ and $d_{i, l}$ denote the dead zone width parameters. In this paper, we assume that the output of the dead zone is unmeasurable, and the dead zone parameters $m_{i, r}, m_{i, l}, d_{i, r}$, and $d_{i, l}$ are not available, but their signs are available $\left(m_{i, r}>0, m_{i, l}>0, d_{i, r} \geq 0\right.$, and $d_{i, l} \geq 0$, respectively). Dead-zone slopes are bounded by known constants $m_{i, r \max }, m_{i, r \min }, m_{i, l \max }$ and $m_{i, l \text { min }}$ such that $0<m_{i, r \min } \leq m_{i, r} \leq m_{i, r \max }$ and $0<m_{i, l \min } \leq m_{i, l} \leq m_{i, l \max }$. The dead-zone inverse technique is used to compensate the dead-zone effect [7]. Setting $u_{i}^{*}$ as the control input which is free of a dead zone, the control signal $u_{i}$ can be expressed as follows:

$$
u_{i}=\left[D_{i}\left(u_{i}^{*}\right)\right]^{-1}=\frac{u_{i}^{*}+\widehat{d}_{i, r, m}}{\widehat{m}_{i, r}} \delta_{i}+\frac{u_{i}^{*}+\widehat{d}_{i, l, m}}{\widehat{m}_{i, l}}\left(1-\delta_{i}\right),
$$

where $\widehat{m}_{i, r}, \widehat{m}_{i, l}, \widehat{d}_{i, r, m}$, and $\widehat{d}_{i, r, l}$ are the estimations of $m_{i, r}$, $m_{i, l}, m_{i, r} d_{i, r}$, and $m_{i, l} d_{i, l}$, respectively. And,

$$
\delta_{i}= \begin{cases}1, & \text { if } u_{i}^{*} \geq 0 \\ 0, & \text { if } u_{i}^{*} \leq 0\end{cases}
$$

The resulting error between $u_{i}^{*}$ and $u_{i}$ is given by

$$
\begin{aligned}
D_{i}\left(u_{i}\right)-u_{i}^{*}= & \left(\tilde{d}_{i, r, m}-\frac{u_{i}^{*}+\widehat{d}_{i, r, m}}{\widehat{m}_{i, r}} \tilde{m}_{i, r}\right) \delta_{i} \\
& +\left(\tilde{d}_{i, l, m}-\frac{u_{i}^{*}+\widehat{d}_{i, l, m}}{\widehat{m}_{i, l}} \tilde{m}_{i, l}\right)\left(1-\delta_{i}\right)+\varepsilon_{i_{u_{i}^{*}}},
\end{aligned}
$$

where parameter errors are defined as $\widetilde{m}_{i, r}=m_{i, r}-\widehat{m}_{i, r}$, $\tilde{m}_{i, l}=m_{i, l}-\widehat{m}_{i, l}, \quad \widetilde{d}_{i, r, m}=d_{i, r, m}-\widehat{d}_{i, r, m}, \quad$ and $\tilde{d}_{i, r, l}=$ $d_{i, l, m}-\widehat{d}_{i, l, m}$.
The bound $\varepsilon_{i_{u_{i}^{*}}}$ is expressed as

$$
\varepsilon_{i_{u_{i}^{*}}}=-m_{i, r} \kappa_{i, r}\left(u_{i}-d_{i, r}\right)-m_{i, l} \kappa_{i, l}\left(u_{i}-d_{i, l}\right),
$$

where

$$
\begin{aligned}
& \kappa_{i, r}= \begin{cases}1, & \text { if } 0 \leq u_{i}<d_{i, r} \\
0, & \text { otherwise, }\end{cases} \\
& \kappa_{i, l}= \begin{cases}1, & \text { if } d_{i, l} \leq u_{i}<0, \\
0, & \text { otherwise. }\end{cases}
\end{aligned}
$$

To solve the state-constrained problem as well as removing the restriction of the feasibility condition, a nonlinear state-dependent function is introduced, which has form below:

$$
\chi_{i, j}=\log \frac{\lambda_{i, j, 1}+x_{i, j}}{\lambda_{i, j, 2}-x_{i, j}}
$$

where $\lambda_{i, j, 1}$ and $\lambda_{i, j, 2}$ are time-varying bounded functions and $\chi_{i, j}$ is the state which is constrained.

From (8), $\chi_{i, j}$ holds the following property:

$$
\begin{aligned}
\chi_{i, j} & \longrightarrow \pm \infty, \quad \text { only if } x_{i, j} \longrightarrow \lambda_{i, j, 2} \\
\text { or } x_{i, j} & \longrightarrow-\lambda_{i, j, 1} .
\end{aligned}
$$

The property means that the state $x_{i, j}$ will be constrained in the region $\left(-\lambda_{i, j, 1}, \lambda_{i, j, 2}\right)$ only when $\chi_{i, j}$ is bounded.

In order to complete the constrained control design without the feasibility condition, system (1) will be transformed into a brand-new system without any constraint. From (6), we can obtain

$$
\begin{aligned}
x_{i, j} & =\frac{e^{\chi_{i, j}} \lambda_{i, j, 2}-\lambda_{i, j, 1}}{e^{\chi_{i, j}}+1}, \\
\mathrm{~d} x_{i, j} & =\frac{e^{\chi_{i, j}} \dot{\lambda}_{i, j, 2}-\dot{\lambda}_{i, j, 1}}{e^{\chi_{i, j}}+1}+\frac{e^{\chi_{i, j}}\left(\lambda_{i, j, 2}+\lambda_{i, j, 1}\right)}{\left(e^{\chi_{i, j}}+1\right)^{2}} \mathrm{~d} \chi_{i, j} .
\end{aligned}
$$

Substituting (10) and (11) into system (1), a new system dynamic is obtained as

$$
\sum_{i}\left\{\begin{array}{l}
\mathrm{d} \chi_{i, 1}=\left[h_{i, 1}\left(\chi_{i, 2}+f_{i, 1}\left(\chi_{i}\right)+\Delta_{i, 1}(\bar{Y})\right)+l_{i, 1}\right] \mathrm{d} t+\bar{g}_{i, 1}\left(\chi_{i}\right) \mathrm{d} w \\
\mathrm{~d} \chi_{i, 2}=\left[h_{i, 2}\left(\chi_{i, 3}+f_{i, 2}\left(\chi_{i}\right)+\Delta_{i, 2}(\bar{Y})\right)+l_{i, 2}\right] \mathrm{d} t+\bar{g}_{i, 2}\left(\chi_{i}\right) \mathrm{d} w \\
\vdots \\
\mathrm{d} \chi_{i, n_{i}-1}=\left[h_{i, n_{i}-1}\left(\chi_{i, n_{i}}+f_{i, n_{i}-1}\left(\chi_{i}\right)+\Delta_{i, n_{i}-1}(\bar{Y})\right)+l_{i, n_{i}-1}\right] \mathrm{d} t+\bar{g}_{i, n_{i}-1}\left(\chi_{i}\right) \mathrm{d} w \\
\mathrm{~d} \chi_{i, n_{i}}=\left[h_{i, n_{i}}\left(D_{i}\left(u_{i}\right)+f_{i, n_{i}}\left(\chi_{i}\right)+\Delta_{i, n_{i}}(\bar{Y})\right]+l_{i, n_{i}}\right] \mathrm{d} t+\bar{g}_{i, n_{i}}\left(\chi_{i}\right) \mathrm{d} w \\
Y_{i}=\chi_{i, 1},
\end{array}\right.
$$


where

$$
\begin{aligned}
\chi_{i} & =\left[\chi_{i, 1}, \chi_{i, 2}, \ldots, \chi_{i, n_{i}}\right]^{T}, \\
l_{i, j} & =-\frac{\left(e^{\chi_{i, j}}+1\right)^{2}\left(e^{\chi_{i, j}} \dot{i}_{i, j, 2}-\dot{\lambda}_{i, j, 1}\right)}{e^{\chi_{i, j}}\left(\lambda_{i, j, 2}+\lambda_{i, j, 1}\right)\left(e^{\chi_{i, j}}+1\right)}, \\
\bar{g}_{i, j} & =-\frac{\left(e^{\chi_{i, j}}+1\right)^{2}}{e^{\chi_{i, j}}\left(\lambda_{i, j, 2}+\lambda_{i, j, 1}\right)} g_{i, j}, \\
h_{i, j} & =\frac{\left(e^{\chi_{i, j}}+1\right)^{2}}{e^{\chi_{i, j}}\left(\lambda_{i, j, 2}+\lambda_{i, j, 1}\right)}, \\
\bar{Y} & =\left[Y_{1}, Y_{2}, \ldots Y_{N}\right]^{T} .
\end{aligned}
$$

Remark 2. Although BLF is regarded as a strong tool for constrained issues, some limitations exist. The virtual controllers are required to meet the feasibility conditions, which means the optimal parameters have to be selected offline and additional computation is inevitable. By utilizing the above system transformation, the constrained closedloop system is transformed into a novel closed-loop system without any constraint. The control design will become easier, and the control design for the novel closed-loop system can achieve the same control objective.

Assumption 1. The unknown nonlinear function $\Delta_{i, j}(\bar{Y}), i=$ $1,2, \ldots, N$ and $j=1,2, \ldots, n_{i}$, satisfies the following inequality, which is a common assumption for large-scale systems:

$$
\left|\Delta_{i, j}(\bar{Y})\right| \leq \sum_{l=1}^{N} \rho_{i, j, l}^{2}\left(Y_{l}\right)
$$

where $\rho_{i, j, l}$ is an unknown smooth function satisfies $\rho_{i, j, l}(0)=0$.

Since system (1) contains unknown nonlinear dynamics, the FLSs are employed to approximate these unknown nonlinear dynamics.

For any continuous function $\rho(v)$ defined over a compact set $\Lambda$ and any given positive constant $\psi$, there always exists an FLS $\hat{\rho}\left(v \mid \mu^{*}\right)=\mu^{* T} \phi(v)$ such that

$$
\sup _{v \in \Lambda}\left|\rho(v)-\mu^{* T} \phi(v)\right| \leq \psi \text {, }
$$

where $\phi_{i}(v)$ are always chosen as the Gaussian functions. $\phi(v)=\left[\phi_{1}(v), \phi_{2}(v), \ldots, \phi_{N}(v)\right]^{T} / \sum_{1}^{N} \phi_{i}(v)$ are the fuzzy basis function vectors and satisfy $0<\phi^{T}(v) \phi(v) \leq 1$. The ideal weight is defined as $\rho=\left[\rho_{1}, \rho_{2}, \ldots, \rho_{N}\right]^{T}$, and $M$ is the fuzzy rule numbers.

\section{Robust Constrained Control Design}

In this section, we will carry out the control design for new system (12) with the new state vector $\chi_{i}$ and new nonlinear functions $h_{i, j}, f_{i, j}, l_{i, j}$, and $\bar{g}_{i, j}$. Combining backstepping design with the dead zone inverse technique, an adaptive fuzzy decentralized controller is established, and based on the Lyapunov stability theory, the stability of the closed-loop system is proved. The $n_{i}$ steps backstepping control design process is based on the following coordinate transformation:

$$
\begin{aligned}
& z_{i, 1}=Y_{i}-Y_{i, r} \\
& z_{i, j}=\chi_{i, j}-\alpha_{i, j-1}, \quad j=2,3, \ldots, n_{i},
\end{aligned}
$$

where $Y_{i, r}=\log \left(\left(\lambda_{i, 1,1}+y_{i, r}\right) /\left(\lambda_{i, 1,2}-y_{i, r}\right)\right)$ is the new reference signal with $y_{i, r}$ being the original reference signal, $z_{i, j}$ denotes the tracking error, and $\alpha_{i, j}$ represents the virtual control signal which will be designed in each step.

Step 1: from (12) and (16), the derivative of $z_{i, 1}$ is expressed as

$$
\mathrm{d} z_{i, 1}=\left[h_{i, 1}\left(\chi_{i, 2}+f_{i, 1}\left(\chi_{i}\right)+\Delta_{i, 1}(\bar{Y})\right)+l_{i, 1}-\dot{Y}_{i, r}\right] \mathrm{d} t+\bar{g}_{i, 1}\left(\chi_{i}\right) \mathrm{d} w .
$$

Consider the following Lyapunov function candidate:

$$
V_{1}=\sum_{i=1}^{N} V_{i, 1}=\sum_{i=1}^{N}\left(\frac{1}{4} z_{i, 1}^{4}+\frac{1}{2 \gamma_{i, 1}} \widetilde{\Theta}_{i, 1}^{2}+\frac{1}{2 \varsigma_{i}} \widetilde{W}_{i}^{2}\right),
$$

where $\gamma_{i, 1}>0$ is a design parameter, $\widetilde{\Theta}_{i, j}=\Theta_{i, j}-\widehat{\Theta}_{i, j}, \widehat{\Theta}_{i, j}$ is the estimation of $\Theta_{i, j}, \Theta_{i, j}=\left\|\theta_{i, j}\right\|^{2}, \widetilde{W}_{i}=W_{i}-\widehat{W}_{i}, W_{i}$ is the estimation of $W_{i}$, and $W_{i}=\left\|w_{i}\right\|^{4}$. The definitions of $w_{i}$ and $\theta_{i, j}$ will be given later.

Combining (17) and (18), it can be obtained that

$$
\begin{aligned}
\ell V_{1} \leq & \sum_{i=1}^{N}\left\{z _ { i , 1 } ^ { 3 } \left[h_{i, 1}\left(z_{i, 2}+\alpha_{i, 1}+f_{i, 1}\left(\chi_{i}\right)+\Delta_{i, 1}(\bar{Y})\right)\right.\right. \\
& \left.\left.+l_{i, 1}-\dot{Y}_{i, r}\right]+\frac{3}{2} z_{i, 1}^{2} \bar{g}_{i, 1}^{T} \bar{g}_{i, 1}-\frac{1}{\gamma_{i, 1}} \widetilde{\Theta}_{i, 1} \dot{\hat{\Theta}}_{i, 1}-\frac{1}{\varsigma_{i}} \widetilde{W}_{i} \dot{\hat{W}}_{i}\right\} .
\end{aligned}
$$

Based on Young's inequality, we have

$$
\begin{aligned}
& z_{i, 1}^{3} h_{i, 1} z_{i, 2} \leq \frac{1}{4} z_{i, 2}^{4}+\frac{3}{4} z_{i, 1}^{4} h_{i, 1}^{4 / 3}, \\
& \frac{3}{2} z_{i, 1}^{2} \bar{g}_{i, 1}^{T} \bar{g}_{i, 1} \leq \frac{3}{4} z_{i, 1}^{4}\left\|\bar{g}_{i, 1}\right\|^{4}+\frac{3}{4}, \\
& \sum_{i=1}^{N} z_{i, 1}^{3} \Delta_{i, 1}(\bar{Y}) \leq \sum_{i=1}^{N} \frac{1}{2} z_{i, 1}^{6}+\sum_{i=1}^{N} Y_{i}^{2} \sum_{l=1}^{N} \frac{1}{2} \bar{\rho}_{l, 1, i}^{2}\left(Y_{i}\right) .
\end{aligned}
$$

Substituting (20)-(22) into (19) yields

$$
\begin{aligned}
\ell V_{1} \leq & \sum_{i=1}^{N}\left[z_{i, 1}^{3}\left(h_{i, 1} \alpha_{i, 1}+\bar{f}_{i, 1}\right)+Y_{i} F_{i}-\frac{1}{\varsigma_{i}} \widetilde{W}_{i} \dot{\hat{W}}+\frac{1}{4} z_{i, 2}^{4}\right. \\
& \left.+\frac{3}{4}-\frac{1}{\gamma_{i, 1}} \widetilde{\Theta}_{i, 1} \dot{\hat{\Theta}}_{i, 1}-Y_{i}^{2} \sum_{l=1}^{N} \sum_{j=1}^{n_{l}} \sum_{k=1}^{j} \frac{1}{2} \bar{\rho}_{l, k, i}^{2}\left(Y_{i}\right)\right],
\end{aligned}
$$

where $\bar{f}_{i, 1}=h_{i, 1} f_{i, 1}\left(\chi_{i}\right)+(3 / 4) z_{i, 1} h_{i, 1}^{4 / 3}+(1 / 2) z_{i, 1}^{3}+(3 / 4)$ $z_{i, 1}^{3} \bar{g}_{i, 1}^{4}+l_{i, 1}-\dot{Y}_{i, r}$ and $F_{i}=Y_{i} \sum_{l=1}^{N} \sum_{j=1}^{n_{l}} \sum_{k=1}^{j}(1 / 2) \bar{\rho}_{l, k, i}^{2}\left(Y_{\underline{i}}\right)$.

Then, the following FLSs are used to approximate $\bar{f}_{i, 1}$ and $F_{i}$ as 


$$
\begin{gathered}
\bar{f}_{i, 1}\left(X_{i, 1}\right)=\theta_{i, 1}^{T} \varphi_{i, 1}\left(X_{i, 1}\right)+\varepsilon_{i, 1}, \\
F_{i}\left(Y_{i}\right)=w_{i}^{T} \phi_{i}\left(Y_{i}\right)+\delta_{i},
\end{gathered}
$$

where the unknown constants $\varepsilon_{i, 1}$ and $\delta_{i}$ denote the approximated errors and satisfy $\left|\varepsilon_{i, 1}\right| \leq \varepsilon_{i, 1}^{*}, \quad\left|\delta_{i}\right| \leq \delta_{i}^{*}$. $X_{i, 1}=\left(\chi_{i}, Y_{i, r}\right)$.

Substituting (24) and (25) into (23) yields

$$
\begin{aligned}
\ell V_{1} \leq & \sum_{i=1}^{N}\left[z_{i, 1}^{3}\left(h_{i, 1} \alpha_{i, 1}+\theta_{i, 1}^{T} \varphi_{i, 1}\left(X_{i, 1}\right)+\varepsilon_{i, 1}\right)+\frac{1}{4} z_{i, 2}^{4}\right. \\
& \left.\quad-Y_{i}^{2} \sum_{l=1}^{N} \sum_{j=1}^{n_{l}} \sum_{k=1}^{j} \frac{1}{2} \bar{\rho}_{l, k, i}^{2}\left(Y_{i}\right)+Y_{i}\left(w_{i}^{T} \phi_{i}\left(Y_{i}\right)+\delta_{i}\right)-\frac{1}{\gamma_{i, 1}} \widetilde{\Theta}_{i, 1} \dot{\Theta}_{i, 1}-\frac{1}{\varsigma_{i}} \widetilde{W}_{i} \dot{\hat{W}}+\frac{3}{4}\right] .
\end{aligned}
$$

By completing the squares, one has

$$
\begin{aligned}
z_{i, 1}^{3}\left(\theta_{i, 1}^{T} \varphi_{i, 1}\left(X_{i, 1}\right)+\varepsilon_{i, 1}\right) & \leq \frac{z_{i, 1}^{6} \Theta_{i, 1}}{4 \tau_{i} \varphi_{i, 1}^{T}\left(\bar{X}_{i, 1}\right) \varphi_{i, 1}\left(\bar{X}_{i, 1}\right)}+\tau_{i}\left(1+\varepsilon_{i, 1}^{* 2}\right)+\frac{z_{i, 1}^{6}}{4 \tau_{i}}, \\
Y_{i}\left(w_{i}^{T} \phi_{i}\left(Y_{i}\right)+\delta_{i}\right) & \leq \frac{z_{i, 1}^{4} W_{i}}{4 \tau_{i}}+\frac{z_{i, 1}^{4}}{4 \tau_{i}}+\frac{3 \tau_{i}}{4}\left(1+2\left\|\delta_{i}^{*}\right\|^{4 / 3}\right)+\frac{W_{i}}{4 \tau_{i}}+\frac{3 \tau_{i}}{4} Y_{i, r}^{2}+\frac{Y_{i, r}^{4}}{4 \tau_{i}},
\end{aligned}
$$

where $\bar{X}_{i, 1}=\left(\chi_{i, 1}, Y_{i, r}\right)$.

$$
\begin{array}{r}
\ell V_{1} \leq \sum_{i=1}^{N}\left[z _ { i , 1 } ^ { 3 } \left(h_{i, 1} \alpha_{i, 1}+\frac{z_{i, 1}^{3} \widehat{\Theta}_{i, 1}}{4 \tau_{i} \varphi_{i, 1}^{T}\left(\bar{X}_{i, 1}\right) \varphi_{i, 1}\left(\bar{X}_{i, 1}\right)}\right.\right. \\
-Y_{i}^{2} \sum_{l=1}^{N} \sum_{j=1}^{n_{l}} \sum_{k=1}^{j} \frac{1}{2} \bar{\rho}_{l, k, i}^{2}\left(Y_{i}\right)+\frac{1}{\gamma_{i, 1}} \widetilde{\Theta}_{i, 1}\left(\frac{}{4 \tau_{i}}\right)^{-} \\
D_{i, 1}=\tau_{i}\left(1+\varepsilon_{i, 1}^{* 2}\right)+\frac{3 \tau_{i}}{4}\left(1+2\left\|\delta_{i}^{*}\right\|^{4 / 3}\right) \\
+\frac{W_{i}}{4 \tau_{i}}+\frac{3 \tau_{i}}{4} Y_{i, r \max }^{2}+\frac{Y_{i, r \max }^{4}}{4 \tau_{i}}+\frac{3}{4}
\end{array}
$$

Design the virtual control function $\alpha_{i, 1}$ and adaptive laws of $\widehat{\Theta}_{i, 1}$ and $\widehat{W}_{i}$ as below:

$$
\begin{aligned}
& \alpha_{i, 1}=h_{i, 1}^{-1}\left[-c_{i, 1} z_{i, 1}-\frac{z_{i, 1}^{3} \widehat{\Theta}_{i, 1}}{4 \tau_{i} \varphi_{i, 1}^{T}\left(\bar{X}_{i, 1}\right) \varphi_{i, 1}\left(\bar{X}_{i, 1}\right)}-\frac{z_{i, 1} \widehat{W}_{i}}{4 \tau_{i}}-\frac{z_{i, 1}^{3}+z_{i, 1}}{4 \tau_{i}}\right], \\
& \dot{\widehat{\Theta}}_{i, 1}=\frac{\gamma_{i, 1} z_{i, 1}^{6}}{4 \tau_{i} \varphi_{i, 1}^{T}\left(\bar{X}_{i, 1}\right) \varphi_{i, 1}\left(\bar{X}_{i, 1}\right)}-\sigma_{i, 1} \widehat{\Theta}_{i, 1},
\end{aligned}
$$

Substituting (31)-(33) into (29) yields

$$
\begin{aligned}
\ell V_{1} \leq & \sum_{i=1}^{N}\left[-c_{i, 1} z_{i, 1}^{4}-Y_{i}^{2} \sum_{l=1}^{N} \sum_{j=1}^{n_{l}} \sum_{k=1}^{j} \frac{1}{2} \bar{\rho}_{l, k, i}^{2}\left(Y_{i}\right)+\frac{1}{4} z_{i, 2}^{4}\right. \\
& \left.+\frac{\sigma_{i, 1}}{\gamma_{i, 1}} \widetilde{\Theta}_{i, 1} \widehat{\Theta}_{i, 1}+\frac{\bar{\sigma}_{i}}{\varsigma_{i}} \widetilde{W}_{i} \widehat{W}+D_{i, 1}\right] .
\end{aligned}
$$

Step $i$ and $j\left(1 \leq i \leq N\right.$ and $\left.2 \leq j \leq n_{i}\right)$ : based on (12) and (16), the derivative of $z_{i, j}$ is expressed as

$$
\begin{aligned}
\mathrm{d} z_{i, j}= & \left\{h_{i, j}\left[\chi_{i, j+1}+f_{i, j}\left(\chi_{i}\right)+\Delta_{i, j}(\bar{Y})\right]+l_{i, j}-L \alpha_{i, j}\right. \\
& \left.-\sum_{k=1}^{j-1} \frac{\alpha_{i, j-1}}{\chi_{i, k}} h_{i, k} \Delta_{i, k}(\bar{Y})\right\} \mathrm{d} t+\bar{g}_{i, j}\left(\chi_{i}\right) \mathrm{d} w,
\end{aligned}
$$


6

Complexity

where

$$
\begin{aligned}
L \alpha_{i, j}= & \sum_{k=1}^{j-1} \frac{\partial \alpha_{i, j-1}}{\partial \chi_{i, k}}\left[h_{i, k}\left(\chi_{i, k+2}+f_{i, k}\left(\chi_{i}\right)\right)+l_{i, k}\right] \\
& +\sum_{k=1}^{j-1} \frac{\partial \alpha_{i, j-1}}{\partial Y_{i, r}^{(k)}} Y_{i, r}^{(k+1)}+\sum_{k=1}^{i-1} \frac{\partial \alpha_{i, j-1}}{\widehat{\Theta}_{i, k}} \dot{\widehat{\Theta}}_{i, k}+\frac{\partial \alpha_{i, j-1}}{\partial \widehat{W}_{i}} \dot{\widehat{W}}_{i} \\
& +\frac{1}{2} \sum_{p_{1}, p_{2}=1}^{j-1} \frac{\partial^{2} \alpha_{i, j-1}}{\partial \chi_{p_{1}} \partial \chi_{p_{2}}} \bar{g}_{i, p_{1}} \bar{g}_{i, p_{2}}^{T} .
\end{aligned}
$$

Consider the following Lyapunov function candidate:

$$
V_{j}=\sum_{i=1}^{N} V_{i, j}=\sum_{i=1}^{N}\left(V_{i, j-1}+z_{i, j}^{4}+\frac{1}{2 \gamma_{i, j}} \widetilde{\Theta}_{i, j}^{2}\right),
$$

where $\gamma_{i, j}>0$ is a design parameter.

Combining (35) and (37), it is shown that

$$
\begin{aligned}
\ell V_{j} \leq & \sum_{i=1}^{N}\left\{-\sum_{k=1}^{j-1} c_{i, k} z_{i, k}^{4}-Y_{i}^{2} \sum_{l=1}^{N} \sum_{q=n_{i}-j+1}^{n_{l}} \sum_{k=1}^{q} \frac{1}{2} \bar{\rho}_{l, k, i}^{2}\left(Y_{i}\right)\right. \\
& +\frac{1}{4} z_{i, j}^{4}+\sum_{k=1}^{j-1} \frac{\sigma_{i, k}}{\gamma_{i, k}} \widetilde{\Theta}_{i, k} \widehat{\Theta}_{i, k}+\frac{\bar{\sigma}_{i}}{\varsigma_{i}} \widetilde{W}_{i} \widehat{W}+D_{i, j-1} \\
& +z_{i, j}^{3}\left[h_{i, j}\left(z_{i, j+1}+\alpha_{i, j}+f_{i, j}\left(\chi_{i}\right)+\Delta_{i, j}(\bar{Y})\right)+l_{i, j}\right. \\
& \left.-L \alpha_{i, j}-\sum_{k=1}^{j-1} \frac{\alpha_{i, j-1}}{\chi_{i, k}} h_{i, k} \Delta_{i, k}(\bar{Y})\right]+\frac{3 z_{i, j}^{3}}{2}\left(\bar{g}_{i, j}-\sum_{k=1}^{j-1} \frac{\partial \alpha_{i, j-1}}{\partial \chi_{i, k}} \bar{g}_{i, k}\right) \\
& \cdot\left(\bar{g}_{i, j}-\sum_{k=1}^{j-1} \frac{\partial \alpha_{i, j-1}}{\partial \chi_{i, k}} \bar{g}_{i, k}\right)^{T}-\frac{1}{y_{i, j}} \widetilde{\Theta}_{i, j} \dot{\hat{\Theta}}_{i, j},
\end{aligned}
$$

where $D_{i, j-1}=D_{i, 1}+(3(j-2) / 4)+\sum_{k=2}^{j-1}\left(1+\tau_{i}\right) \varepsilon_{i, k}^{* 2}$.

Based on Young's inequality, we obtain

$$
\begin{aligned}
z_{i, j}^{3} h_{i, j} z_{i, j+1} \leq & \frac{1}{4} z_{i, j+1}^{4}+\frac{3}{4} z_{i, j}^{4} h_{i, j}^{4 / 3}, \\
& \frac{3 z_{i, j}^{3}}{2}\left(\bar{g}_{i, j}-\sum_{k=1}^{j-1} \frac{\partial \alpha_{i, j-1}}{\partial \chi_{i, k}} \bar{g}_{i, k}\right)\left(\bar{g}_{i, j}-\sum_{k=1}^{j-1} \frac{\partial \alpha_{i, j-1}}{\partial \chi_{i, k}} \bar{g}_{i, k}\right)^{T} \\
\leq & \frac{3 z_{i, j}^{6}}{4}\left\|\bar{g}_{i, j}-\sum_{k=1}^{j-1} \frac{\partial \alpha_{i, j}-1}{\partial \chi_{i, k}} \bar{g}_{i, k}\right\|^{4}+\frac{3}{4}, \\
& \sum_{i=1}^{N} z_{i, j}^{3} h_{i, j} \Delta_{i, j}(\bar{Y}) \leq \sum_{i=1}^{N} \frac{1}{2} z_{i, j}^{6} h_{i, j}^{2}+\sum_{i=1}^{N} Y_{i}^{2} \sum_{l=1}^{N} \frac{1}{2} \bar{\rho}_{l, j, i}^{2}\left(Y_{i}\right), \\
& -\sum_{i=1}^{N} z_{i, j}^{3} \sum_{k=1}^{j-1} \frac{\alpha_{i, j-1}}{\chi_{i, k}} h_{i, k} \Delta_{i, k}(\bar{Y}) \leq \sum_{i=1}^{N} \sum_{k=1}^{j-1} \frac{1}{2}\left(\frac{\alpha_{i, j-1}}{\chi_{i, k}}\right)^{2} z_{i, j}^{6} h_{i, k}^{2}+\sum_{i=1}^{N} Y_{i}^{2} \sum_{k=1}^{j-1} \sum_{l=1}^{N} \frac{1}{2} \bar{\rho}_{l, k, i}^{2}\left(Y_{i}\right) .
\end{aligned}
$$

Then, we have

$$
\begin{aligned}
\ell V_{j} \leq & \sum_{i=1}^{N}\left\{-\sum_{k=1}^{j-1} c_{i, k} z_{i, k}^{4}-Y_{i}^{2} \sum_{l=1}^{N} \sum_{q=n_{i}-j+1}^{n_{l}} \sum_{k=1}^{q} \frac{1}{2} \bar{\rho}_{l, k, i}^{2}\left(Y_{i}\right)\right. \\
& +\frac{1}{4} z_{i, j}^{4}+\sum_{k=1}^{j-1} \frac{\sigma_{i, k}}{\gamma_{i, k}} \widetilde{\Theta}_{i, k} \widehat{\Theta}_{i, k}+\frac{\bar{\sigma}_{i}}{\varsigma_{i}} \widetilde{W}_{i} \widehat{W}+\frac{1}{4} z_{i, j+1}^{4} \\
& +\frac{3}{4} z_{i, j}^{4} h_{i, j}^{4 / 3}+z_{i, j}^{3}\left[h_{i, j}\left(\alpha_{i, j}+f_{i, j}\left(\chi_{i}\right)+\Delta_{i, j}(\bar{Y})\right)\right. \\
& \left.+l_{i, j}-L \alpha_{i, j}-\sum_{k=1}^{j-1} \frac{\alpha_{i, j-1}}{\chi_{i, k}} h_{i, k} \Delta_{i, k}(\bar{Y})\right]+\frac{1}{\gamma_{i, j}} \widetilde{\Theta}_{i, j} \dot{\widehat{\Theta}}_{i, j}
\end{aligned}
$$




$$
\begin{aligned}
& \left.+\frac{3 z_{i, j}^{6}}{4}\left\|\bar{g}_{i, j}-\sum_{k=1}^{j-1} \frac{\partial \alpha_{i, j-1}}{\partial \chi_{i, k}} \bar{g}_{i, k}\right\|^{4}+\frac{3}{4}+D_{i, j-1}\right\}, \\
\ell V_{j} \leq & \sum_{i=1}^{N}\left\{-\sum_{k=1}^{j-1} c_{i, k} z_{i, k}^{4}-Y_{i}^{2} \sum_{l=1}^{N} \sum_{q=n_{i}-j+1}^{n_{l}} \sum_{k=1}^{q} \frac{1}{2} \bar{\rho}_{l, k, i}^{2}\left(Y_{i}\right)\right. \\
& +\frac{1}{4} z_{i, j}^{4}+\sum_{k=1}^{j-1} \frac{\sigma_{i, k}}{\gamma_{i, k}} \widetilde{\Theta}_{i, k} \widehat{\Theta}_{i, k}+\frac{\bar{\sigma}_{i}}{\varsigma_{i}} \widetilde{W}_{i} \widehat{W}+\frac{1}{4} z_{i, j+1}^{4} \\
& +\frac{3}{4} z_{i, j}^{4} h_{i, j}^{4 / 3}+\frac{1}{2} z_{i, j}^{6} h_{i, j}^{2}+Y_{i}^{2} \sum_{l=1}^{N} \frac{1}{2} \bar{\rho}_{l, j, i}^{2}\left(Y_{i}\right)+\frac{3}{4} \\
& +z_{i, j}^{3}\left[h_{i, j}\left(\alpha_{i, j}+f_{i, j}\left(\chi_{i}\right)\right)+l_{i, j}-L \alpha_{i, j}\right. \\
& \left.\left.-\sum_{k=1}^{j-1} \frac{\alpha_{i, j-1}}{\chi_{i, k}} h_{i, k} \Delta_{i, k}(\bar{Y})\right]+\frac{1}{\gamma_{i, j}} \widetilde{\Theta}_{i, j} \dot{\Theta}_{i, j}+\frac{3 z_{i, j}^{6}}{4}\left\|\bar{g}_{i, j}-\sum_{k=1}^{j-1} \frac{\partial \alpha_{i, j-1}}{\partial \chi_{i, k}} \bar{g}_{i, k}\right\|^{4}+D_{i, j-1}\right\} .
\end{aligned}
$$

We finally yield

$$
\begin{aligned}
\ell V_{j} \leq & \sum_{i=1}^{N}\left[-\sum_{k=1}^{j-1} c_{i, k} z_{i, k}^{4}-Y_{i}^{2} \sum_{l=1}^{N} \sum_{q=n_{i}-j}^{n_{l}} \sum_{k=1}^{q} \frac{1}{2} \bar{\rho}_{l, k, i}^{2}\left(Y_{i}\right)\right. \\
& +\frac{1}{4} z_{i, j+1}^{4}+\sum_{k=1}^{j-1} \frac{\sigma_{i, k}}{\gamma_{i, k}} \widetilde{\Theta}_{i, k} \widehat{\Theta}_{i, k}+\frac{\bar{\sigma}_{i}}{\varsigma_{i}} \widetilde{W}_{i} \widehat{W}+D_{i, j-1}+\frac{3}{4} \\
& \left.+z_{i, j}^{3}\left(h_{i, j} \alpha_{i, j}+\bar{f}_{i, j}\right)-\frac{1}{\gamma_{i, j}} \widetilde{\Theta}_{i, j} \dot{\Theta}_{i, j}\right],
\end{aligned}
$$

where

$$
\begin{aligned}
\bar{f}_{i, j}= & h_{i, j} f_{i, j}\left(\chi_{i}\right)+\frac{3}{4} z_{i, j} h_{i, j}^{4 / 3}+\frac{1}{4} z_{i, j}+\frac{1}{2} z_{i, j}^{3} h_{i, j}^{2} \\
& +\sum_{k=1}^{j-1} \frac{1}{2}\left(\frac{\alpha_{i, j-1}}{\chi_{i, k}}\right)^{2} z_{i, j}^{3} h_{i, k}^{2}+l_{i, j}-L \alpha_{i, j} \\
& +\frac{3 z_{i, j}^{3}}{4}\left\|\bar{g}_{i, j}-\sum_{k=1}^{j-1} \frac{\partial \alpha_{i, j-1}}{\partial \chi_{i, k}} \bar{g}_{i, k}\right\|^{4} .
\end{aligned}
$$

$$
\begin{aligned}
z_{i, j}^{3}\left[\theta_{i, j}^{T} \varphi_{i, j}\left(X_{i, j}\right)+\varepsilon_{i, j}\right] & =z_{i, j}^{3} \theta_{i, j}^{T} \varphi_{i, j}\left(X_{i, j}\right)+z_{i, j}^{3} \varepsilon_{i, j} \\
& \leq \frac{1}{4 \tau_{i} \varphi_{i, j}^{T}\left(\bar{X}_{i, j}\right) \varphi_{i, j}\left(\bar{X}_{i, j}\right)} z_{i, j}^{6}\left\|\theta_{i, j}\right\|^{2}+\tau_{i} \varphi_{i, j}^{T}\left(\bar{X}_{i, j}\right) \varphi_{i, j}\left(\bar{X}_{i, j}\right)+\tau_{i} \varepsilon_{i, j}^{* 2}+\frac{z_{i, j}^{6}}{4 \tau_{i}} .
\end{aligned}
$$


Then, we obtain

$$
\begin{aligned}
z_{i, j}^{3}\left[\theta_{i, j}^{T} \varphi_{i, j}\left(X_{i, j}\right)+\varepsilon_{i, j}\right] \leq & \frac{z_{i, j}^{6} \Theta_{i, j}}{4 \tau_{i} \varphi_{i, j}^{T}\left(\bar{X}_{i, j}\right) \varphi_{i, j}\left(\bar{X}_{i, j}\right)} \\
& +\tau_{i}\left(1+\varepsilon_{i, j}^{* 2}\right)+\frac{z_{i, j}^{6}}{4 \tau_{i}}
\end{aligned}
$$

where $\bar{X}_{i, j}=\left(\bar{\chi}_{i, j}, \widehat{W}_{i}, \widehat{\Theta}_{i, 1}, \ldots, \widehat{\Theta}_{i, j-1}, Y_{i, r}, Y_{i . r}^{(1)}, \ldots, Y_{i . r}^{(j)}\right)$.

Combining (44) and (46), we obtain

$$
\begin{aligned}
\ell V_{j} \leq & \sum_{i=1}^{N}\left\{-\sum_{k=1}^{j-1} c_{i, k} z_{i, k}^{4}-Y_{i}^{2} \sum_{l=1}^{N} \sum_{q=n_{i}-j}^{n_{l}} \sum_{k=1}^{q} \frac{1}{2} \bar{\rho}_{l, k, i}^{2}\left(Y_{i}\right)\right. \\
& +\frac{1}{4} z_{i, j+1}^{4}+\sum_{k=1}^{j-1} \frac{\sigma_{i, k}}{\gamma_{i, k}} \widetilde{\Theta}_{i, k} \widehat{\Theta}_{i, k}+\frac{\bar{\sigma}_{i}}{\varsigma_{i}} \widetilde{W}_{i} \widehat{W}_{i}+z_{i, j}^{3}\left[h_{i, j} \alpha_{i, j}+\frac{z_{i, j}^{3} \widehat{\Theta}_{i, j}}{4 \tau_{i} \varphi_{i, j}^{T}\left(\bar{X}_{i, j}\right) \varphi_{i, j}\left(\bar{X}_{i, j}\right)}+\frac{z_{i, j}^{3}}{4 \tau_{i}}\right] \\
& \left.+\frac{1}{\gamma_{i, j}} \widetilde{\Theta}_{i, j}\left[\frac{\gamma_{i, j} z_{i, j}^{6}}{4 \tau_{i} \varphi_{i, j}^{T}\left(\bar{X}_{i, j}\right) \varphi_{i, j}\left(\bar{X}_{i, j}\right)}-\dot{\widehat{\Theta}}_{i, j}\right]+D_{i, j}\right\},
\end{aligned}
$$

where $D_{i, j}=D_{i, j-1}+\tau_{i}\left(1+\varepsilon_{i, j}^{* 2}\right)+(3 / 4)$.

Design the virtual controller $\alpha_{i, j}$ and adaptive law of $\widehat{\Theta}_{i, j}$ as

$$
\begin{aligned}
& \alpha_{i, j}=h_{i, j}^{-1}\left[-c_{i, j} z_{i, j}-\frac{z_{i, j}^{3} \widehat{\Theta}_{i, j}}{4 \tau_{i} \varphi_{i, j}^{T}\left(\bar{X}_{i, j}\right) \varphi_{i, j}\left(\bar{X}_{i, j}\right)}-\frac{z_{i, j}^{3}}{4 \tau_{i}}\right], \\
& \dot{\widehat{\Theta}}_{i, j}=\frac{\gamma_{i, j} z_{i, j}^{6}}{4 \tau_{i} \varphi_{i, j}^{T}\left(\bar{X}_{i, j}\right) \varphi_{i, j}\left(\bar{X}_{i, j}\right)}-\sigma_{i, j} \widehat{\Theta}_{i, j} .
\end{aligned}
$$

Substituting (48) and (49) into (41) yields

$$
\begin{aligned}
\ell V_{j} \leq & \sum_{i=1}^{N}\left[-\sum_{k=1}^{j} c_{i, k} z_{i, k}^{4}-Y_{i}^{2} \sum_{l=1}^{N} \sum_{q=n_{i}-j}^{n_{l}} \sum_{k=1}^{q} \frac{1}{2} \bar{\rho}_{l, k, i}^{2}\left(Y_{i}\right)\right. \\
& \left.+\frac{1}{4} z_{i, j+1}^{4}+\sum_{k=1}^{j} \frac{\sigma_{i, k}}{\gamma_{i, k}} \widetilde{\Theta}_{i, k} \widehat{\Theta}_{i, k}+\frac{\bar{\sigma}_{i}}{\varsigma_{i}} \widetilde{W}_{i} \widehat{W}_{i}+D_{i, j}\right] .
\end{aligned}
$$

Step $i$ and $n_{i}$ : in the final step, the actual controller is constructed. Similar to the above steps, the derivative of $z_{i, n_{i}}$ is expressed as

$$
\begin{aligned}
\mathrm{d} z_{i, n_{i}}= & \left\{h_{i, n_{i}}\left[D_{i}\left(u_{i}\right)+f_{i, n_{i}}\left(\chi_{i}\right)+\Delta_{i, n_{i}}(\bar{Y})\right]+l_{i, n_{i}}\right. \\
& \left.-L \alpha_{i, n_{i}}-\sum_{k=1}^{n_{i}-1} \frac{\alpha_{i, n_{i}-1}}{\chi_{i, k}} h_{i, k} \Delta_{i, k}(\bar{Y})\right\} \mathrm{d} t+\bar{g}_{i, n_{i}}\left(\chi_{i}\right) \mathrm{d} w,
\end{aligned}
$$

where

$$
\begin{aligned}
L \alpha_{i, n_{i}}= & \sum_{k=1}^{n_{i}-1} \frac{\partial \alpha_{i, n_{i}-1}}{\partial \chi_{i, k}}\left\{h_{i, k}\left[\chi_{i, k+2}+f_{i, k}\left(\chi_{i}\right)\right]+l_{i, k}\right\} \\
& +\sum_{k=1}^{n_{i}-1} \frac{\partial \alpha_{i, n_{i}-1}}{\partial Y_{i, r}^{(k)}} Y_{i, r}^{(k+1)}+\sum_{k=1}^{n_{i}-1} \frac{\partial \alpha_{i, n_{i}-1}}{\widehat{\Theta}_{i, k}} \dot{\widehat{\Theta}}_{i, k}+\frac{\partial \alpha_{i, n_{i}-1}}{\partial \widehat{\widehat{W}}_{i}} \dot{\widehat{W}}_{i} \\
& +\frac{1}{2} \sum_{p_{1}, p_{2}=1}^{n_{i}-1} \frac{\partial^{2} \alpha_{i, n_{i}-1}}{\partial \chi_{p_{1}} \partial \chi_{p_{2}}} \bar{g}_{i, p_{1}} \bar{g}_{i, p_{2}}^{T} .
\end{aligned}
$$

Consider the following Lyapunov function candidate:

$$
\begin{aligned}
V= & \sum_{i=1}^{N} V_{i, n_{i}}=\sum_{i=1}^{N}\left(V_{i, n_{i}-1}+\frac{1}{4} z_{i, n_{i}}^{4}+\frac{1}{2 \gamma_{i, n_{i}}} \widetilde{\Theta}_{i, n_{i}}^{2},\right. \\
V= & \sum_{i=1}^{N}\left(V_{i, n_{i}-1}+\frac{1}{4} z_{i, n_{i}}^{4}+\frac{1}{2 \gamma_{i, n_{i}}} \widetilde{\Theta}_{i, n_{i}}^{2}+\frac{1}{2 \xi_{i, 1}} \widetilde{m}_{i, r}^{2}\right. \\
& \left.+\frac{1}{2 \xi_{i, 2}} \widetilde{m}_{i, l}^{2}+\frac{1}{2 \xi_{i, 3}} \widetilde{d}_{i, r, m}^{2}+\frac{1}{2 \xi_{i, 4}} \widetilde{d}_{i, l, m}^{2}\right),
\end{aligned}
$$

where $\gamma_{i, n_{i}}>0, \quad \xi_{i, 1}>0, \quad \xi_{i, 2}>0, \quad \xi_{i, 3}>0$, and $\xi_{i, 4}>0$ are designed parameters.

Combining (51) and (53), it can be shown that 


$$
\begin{aligned}
\ell V \leq & \sum_{i=1}^{N}\left\{-\sum_{k=1}^{n_{i}-1} c_{i, k} z_{i, k}^{4}+\sum_{k=1}^{n_{i}-1} \frac{\sigma_{i, k}}{\gamma_{i, k}} \widetilde{\Theta}_{i, k} \widehat{\Theta}_{i, k}+\frac{\bar{\sigma}_{i}}{\varsigma_{i}} \widetilde{W}_{i} \widehat{W}\right. \\
& -\frac{1}{\xi_{i, 1}} \widetilde{m}_{i, r} \dot{\bar{m}}_{i, r}-\frac{1}{\xi_{i, 2}} \widetilde{m}_{i, l} \dot{\vec{m}}_{i, l}-\frac{1}{\xi_{i, 3}} \widetilde{d}_{i, r, m} \dot{\hat{d}}_{i, r, m} \\
& -\frac{1}{\xi_{i, 4}} \widetilde{d}_{i, l, m} \dot{\vec{d}}_{i, l, m}+z_{i, n_{i}}^{3} h_{i, n_{i}}\left[\delta_{i}\left(\widetilde{d}_{i, r, m}-\frac{u_{i}^{*}+\widehat{d}_{i, r, m}}{\widehat{m}_{i, r}} \widetilde{m}_{i, r}\right)\right. \\
& \left.+\left(\widetilde{d}_{i, l, m}-\frac{u_{i}^{*}+\widehat{d}_{i, l, m}}{\widehat{m}_{i, l}} \widetilde{m}_{i, l}\right)\left(1-\delta_{i}\right)\right]+z_{i, n_{i}}^{3}\left[h_{i, n_{i}} u_{i}^{*}+\frac{z_{i, n_{i}}^{3} \widehat{\Theta}_{i, n_{i}}}{4 \tau_{i} \varphi_{i, n_{i}}^{T}\left(\bar{X}_{i, n_{i}}\right) \varphi_{i, n_{i}}\left(\bar{X}_{i, n_{i}}\right)}+\frac{z_{i, n_{i}}^{3}}{4 \tau_{i}}+\frac{1}{2} z_{i, n_{i}}^{3} h_{i, n_{i}}^{2}\right] \\
& +\frac{1}{\gamma_{i, n_{i}}} \widetilde{\Theta}_{i, n_{i}}\left[\frac{\gamma_{i, n_{i}} z_{i, n_{i}}^{6}}{4 \tau_{i} \varphi_{i, n_{i}}^{T}\left(\bar{X}_{i, n_{i}}\right) \varphi_{i, n_{i}}\left(\bar{X}_{i, n_{i}}\right)}-\dot{\widehat{\Theta}}_{i, n_{i}}\right]+D_{i, n_{i}}
\end{aligned}
$$

where $D_{i, n_{i}}=D_{i, n_{i}-1}+\tau_{i}\left(1+\varepsilon_{i, j}^{* 2}\right)+(3 / 4)+(1 / 2)\left(\varepsilon_{i u_{i}^{*}}\right)^{2}$.

Design the actual controller $u_{i}^{*}$ and adaptive laws of $\widehat{\Theta}_{i, n_{i}}$, $\widehat{m}_{i, r}, \widehat{m}_{i, l}, \hat{d}_{i, r, m}$, and $\hat{d}_{i, r, l}$ as

$$
u_{i}^{*}=h_{i, n_{i}}^{-1}\left[-c_{i, n_{i}} z_{i, n_{i}}-\frac{z_{i, n_{i}}^{3} \widehat{\Theta}_{i, n_{i}}}{4 \tau_{i} \varphi_{i, n_{i}}^{T}\left(\bar{X}_{i, n_{i}}\right) \varphi_{i, n_{i}}\left(\bar{X}_{i, n_{i}}\right)}-\frac{z_{i, n_{i}}^{3}}{4 \tau_{i}}-\frac{1}{2} z_{i, n_{i}}^{3} h_{i, n_{i}}^{2}\right],
$$

$$
\begin{aligned}
& \dot{\widehat{\Theta}}_{i, n_{i}}=\frac{\gamma_{i, n_{i}} z_{i, n_{i}}^{6}}{4 \tau_{i} \varphi_{i, n_{i}}^{T}\left(\bar{X}_{i, n_{i}}\right) \varphi_{i, n_{i}}\left(\bar{X}_{i, n_{i}}\right)}-\sigma_{i, n_{i}} \widehat{\Theta}_{i, n_{i}}, \\
& \dot{\hat{m}}_{i, r}=\frac{u_{i}^{*}+\widehat{d}_{i, r, m}}{\widehat{m}_{i, r}} \xi_{i, 1} z_{i, n_{i}}^{3} h_{i, n_{i}} \delta_{i}+\bar{\xi}_{i, 1} \widehat{m}_{i, r}, \\
& \dot{\hat{m}}_{i, l}=\frac{u_{i}^{*}+\widehat{d}_{i, l, m}}{\widehat{m}_{i, l}} \xi_{i, 2} z_{i, n_{i}}^{3} h_{i, n_{i}}\left(1-\delta_{i}\right)+\bar{\xi}_{i, 2} \widehat{m}_{i, l}, \\
& \dot{\hat{d}}_{i, r, m}=-\xi_{i, 3} z_{i, n_{i}}^{3} h_{i, n_{i}} \delta_{i}+\bar{\xi}_{i, 3} \widehat{d}_{i, r, m}, \\
& \dot{\vec{d}}_{i, l, m}=-\xi_{i, 4} z_{i, n_{i}}^{3} h_{i, n_{i}}\left(1-\delta_{i}\right)+\bar{\xi}_{i, 4} \widehat{d}_{i, l, m} .
\end{aligned}
$$

Substituting (55)-(60) into (54) yields

$$
\begin{aligned}
\ell V \leq & \sum_{i=1}^{N}\left(-\sum_{k=1}^{n_{i}} c_{i, k} z_{i, k}^{4}-\sum_{k=1}^{n_{i}} \frac{\sigma_{i, k}}{2 \gamma_{i, k}} \widetilde{\Theta}_{i, k}^{2}-\frac{\bar{\sigma}_{i}}{2 c_{i}} \widetilde{W}_{i}^{2}+D_{i}\right. \\
& \left.-\frac{\bar{\xi}_{i, 1}}{2 \xi_{i, 1}} \widetilde{m}_{i, r}^{2}-\frac{\bar{\xi}_{i, 2}}{2 \xi_{i, 2}} \widetilde{m}_{i, l}^{2}-\frac{\bar{\xi}_{i, 3}}{2 \xi_{i, 3}} \widetilde{d}_{i, r, m}^{2}-\frac{\bar{\xi}_{i, 4}}{2 \xi_{i, 4}} \widetilde{d}_{i, l, m}^{2}\right),
\end{aligned}
$$

where

$$
\begin{aligned}
D_{i}= & D_{i, j}+\sum_{k=1}^{n_{i}} \frac{\sigma_{i, k}}{2 \gamma_{i, k}} \Theta_{i, k}^{2}+\frac{\bar{\sigma}_{i}}{2 \varsigma_{i}} W_{i}^{2}+\frac{\bar{\xi}_{i, 1}}{2 \xi_{i, 1}} m_{i, r}^{2} \\
& +\frac{\bar{\xi}_{i, 2}}{2 \xi_{i, 2}} m_{i, l}^{2}+\frac{\bar{\xi}_{i, 3}}{2 \xi_{i, 3}} d_{i, r, m}^{2}+\frac{\bar{\xi}_{i, 4}}{2 \xi_{i, 4}} d_{i, l, m}^{2} .
\end{aligned}
$$

Define $C_{i}=\min \left\{c_{i, k}, \sigma_{i, k}, \bar{\sigma}_{i}, \bar{\xi}_{i, 1}, \bar{\xi}_{i, 2}, \bar{\xi}_{i, 3}, \bar{\xi}_{i, 4}\right\}$; then, (61) is written as

$$
\ell V \leq \sum_{i=1}^{N}\left(-C_{i} V_{i, n_{i}}+D_{i}\right)
$$

Let $C=\min _{1 \leq i \leq N}\left\{C_{i}\right\}$ and $D=\max _{1 \leq i \leq N}\left\{D_{i}\right\}$; then, (62) becomes

$$
\ell V \leq-C V+D .
$$

We summarize the above robust constrained control design as the following theorem.

Theorem 1. Consider nonlinear system (12); under Assumption 1, the virtual controllers are designed as (31) and (48), the actual controller is designed as (55), and adaptive laws are designed as (32), (33), (49), and (56)-(60); then, the developed control scheme can guarantee that all the signals in the closed-loop system are bounded, and the tracking errors converge to a small neighborhood of the origin.

Proof. Integrating (64) over $[0, t]$ results in

$$
0 \leq E V(t) \leq e^{-C t} E V(0)+\frac{D}{C} .
$$

From (57), we can obtain that all the closed-loop signals $\chi_{i, j}, z_{i, j}, \widehat{\Theta}_{i, j}, \widehat{W}_{i}, \widehat{m}_{i, r}, \widehat{m}_{i, l}, \widehat{d}_{i, r, m}$, and $\widehat{d}_{i, l, m}$ are bounded, $1 \leq i \leq N$ and $1 \leq j \leq n_{i}$. The tracking error satisfies

$$
\left|z_{i, 1}\right| \leq \sqrt{2\left(e^{-C t} V(0)+\frac{D}{C}\right)} .
$$

Furthermore, since the novel closed-loop signal $\chi_{i, j}$ is bounded, according to the definition of the nonlinear statedependent function in (8), the signal $x_{i, j}$ remains in the constrained region $\left(-\lambda_{i, j, 1}, \lambda_{i, j, 2}\right)$. Then, the proof of Theorem 1 has been completed.

Remark 3. The control design in this paper does not depend on the feasibility condition, and it is no need to utilize the BLFs, which can achieve the same control objective. 
Although the results in [21-33] can also solve the state constrained problem, the control designs appear much complicatedly.

\section{Simulation Example}

Consider an example of two inverted pendulums connected by a moving spring mounted on two cars. The input to each pendulum is the torque $u_{i}(i=1,2)$ applied at the pivot point. We define the state vectors as

$$
\begin{aligned}
& \left(x_{1,1}, x_{1,2}\right)^{T}=\left(\theta_{1}, \dot{\theta}_{1}\right)^{T}, \\
& \left(x_{2,1}, x_{2,2}\right)^{T}=\left(\theta_{2}, \dot{\theta}_{2}\right)^{T} .
\end{aligned}
$$

Define $a(t)=\sin (5 t), K_{1}=\sin (2 t), K_{2}=\sin (3 t)+L$, $\beta_{1}=m \sin x_{1,1} / M, \beta_{2}=m \sin x_{2,1} / M$, and $c=m /(m+M)$. The dynamics equations of the inverted two pendulums on cars are introduced in [3]:

$$
\begin{aligned}
& \left\{\begin{array}{l}
\dot{x}_{1,1}=x_{1,2}, \\
\dot{x}_{1,2}=\left\{\frac{g}{c l}-\frac{k a(t)[a(t)-c l]}{c m l^{2}}\right\} x_{1,1}+\frac{u_{1}}{c m l^{2}}+\frac{k a(t)[a(t)-c l]}{c m l^{2}} x_{2,1}+\beta_{1} x_{1,2}^{2} \\
+\frac{k a(t)[a(t)-c l]}{c m l^{2}}\left(K_{1}-K_{2}\right), \\
y_{1}=x_{1,1}, \\
\dot{x}_{2,1}=x_{2,2}, \\
\dot{x}_{2,2}=\left\{\frac{g}{c l}-\frac{k a(t)[a(t)-c l]}{c m l^{2}}\right\} x_{2,1}+\frac{u_{2}}{c m l^{2}}+\frac{k a(t)[a(t)-c l]}{c m l^{2}} x_{1,1}+\beta_{2} x_{2,2}^{2} \\
y_{2}=x_{2,1}, \\
+\frac{k a(t)[a(t)-c l]}{c m l^{2}}\left(K_{2}-K_{1}\right),
\end{array}\right.
\end{aligned}
$$

where $m(\mathrm{~kg}), M(\mathrm{~kg}), l(\mathrm{~m}), L(\mathrm{~m}), k(\mathrm{~N} / \mathrm{m})$, and $g\left(\mathrm{~m} / \mathrm{s}^{2}\right)$ are the mass of the pendulum and the car, the length of the pole, the natural length of the connecting spring, the spring constant, and the gravity constant, respectively. 
Here, both stochastic disturbance and actuator dead zones are considered on the basis of the above systems:

$$
\begin{aligned}
& \left\{\begin{array}{l}
\dot{x}_{1,1}=x_{1,2}, \\
\dot{x}_{1,2}=\left\{\left[\frac{g}{c l}-\frac{k a(t)(a(t)-c l)}{c m l^{2}}\right] x_{1,1}+\frac{D_{1}\left(u_{1}\right)}{c m l^{2}}+\frac{k a(t)[a(t)-c l]}{c m l^{2}} x_{2,1}+\beta_{1} x_{1,2}^{2}\right. \\
\left.+\frac{k a(t)[a(t)-c l]}{c m l^{2}}\left(K_{1}-K_{2}\right)\right\} \mathrm{d} t+g_{1,2}\left(x_{1,1}, x_{1,2}\right) \mathrm{d} w, \\
y_{1}=x_{1,1}, \\
\dot{x}_{2,1}=x_{2,2}, \\
\dot{x}_{2,2}=\left\{\left[\frac{g}{c l}-\frac{k a(t)(a(t)-c l)}{c m l^{2}}\right] x_{2,1}+\frac{D_{2}\left(u_{2}\right)}{c m l^{2}}+\frac{k a(t)[a(t)-c l]}{c m l^{2}} x_{1,1}+\beta_{2} x_{2,2}^{2}\right. \\
\left.+\frac{k a(t)[a(t)-c l]}{c m l^{2}}\left(K_{2}-K_{1}\right)\right\} \mathrm{d} t+g_{2,2}\left(x_{2,1}, x_{2,2}\right) \mathrm{d} w, \\
y_{2}=x_{2,1},
\end{array}\right.
\end{aligned}
$$

where

$$
\begin{aligned}
& g_{1,2}\left(x_{1,1}, x_{1,2}\right)=\cos \left(x_{1,2}\right)-\sin \left(x_{1,1}\right), \\
& g_{2,2}\left(x_{2,1}, x_{2,2}\right)=\sin \left(x_{2,2}\right)-\cos \left(x_{2,1}\right) .
\end{aligned}
$$

$D u_{1}$ and $D u_{2}$ are the outputs of the unknown actuator dead zones with the dead-zone slopes being given as

$$
\begin{aligned}
m_{1, r} & =m_{2, r}=1.1, \\
m_{1, l} & =m_{2, l}=1.7, \\
d_{1, r} & =d_{2, r}=0.3, \\
d_{1, l} & =d_{2, l}=0.4 .
\end{aligned}
$$

The tracking signals are defined as $y_{1, r}=y_{2, r}=0.45 \sin (t)$, and the states $x_{1,1}, x_{1,2}, x_{2,1}$, and $x_{2,2}$ are constrained in the regions:

$$
\begin{aligned}
& \left(-\lambda_{1,1,1}(t), \lambda_{1,1,2}(t)\right), \\
& \left(-\lambda_{1,2,1}(t), \lambda_{1,2,2}(t)\right), \\
& \left(-\lambda_{2,1,1}(t), \lambda_{2,1,2}(t)\right), \\
& \left(-\lambda_{2,2,1}(t), \lambda_{2,2,2}(t)\right),
\end{aligned}
$$

with

$$
\begin{aligned}
& \lambda_{1,1,1}(t)=\lambda_{2,1,1}(t)=0.5-0.4 \sin (t), \\
& \lambda_{1,1,2}(t)=\lambda_{2,1,2}(t)=0.6-0.1 \cos (t), \\
& \lambda_{1,2,1}(t)=\lambda_{2,2,1}(t)=0.4-0.3 \sin (t), \\
& \lambda_{1,2,2}(t)=\lambda_{2,2,2}(t)=0.5+0.1 \cos (t) .
\end{aligned}
$$

From (10)-(12), the novel closed-loop system dynamics are described as 


$$
\begin{aligned}
& \left\{\begin{array}{l}
\mathrm{d} \chi_{1,1}=\left(h_{1,1} \chi_{1,2}+l_{1,1}\right) \mathrm{d} t, \\
\mathrm{~d} \chi_{1,2}=\left\{h _ { 1 , 2 } \left[\left(\frac{g}{c l}-\frac{k a(t)(a(t)-c l)}{c m l^{2}}\right) \chi_{1,1}+\frac{D u_{1}}{c m l^{2}}+\frac{k a(t)(a(t)-c l)}{c m l^{2}} \chi_{2,1}+\bar{\beta}_{1} \chi_{1,2}^{2}\right.\right. \\
\left.\left.+\frac{k a(t)(a(t)-c l)}{c m l^{2}}\left(K_{1}-K_{2}\right)\right]+l_{1,2}\right\} \mathrm{d} t+\bar{g}_{1,2}\left(\chi_{1,1}, \chi_{1,2}\right) \mathrm{d} w \\
Y_{1}=\chi_{1,1}, \\
\mathrm{~d} \chi_{2,1}=\left(h_{2,1} \chi_{2,2}+l_{2,1}\right) \mathrm{d} t, \\
\mathrm{~d} \chi_{2,2}=\left\{h _ { 2 , 2 } \left[\left(\frac{g}{c l}-\frac{k a(t)(a(t)-c l)}{c m l^{2}}\right) \chi_{2,1}+\frac{D u_{2}}{c m l^{2}}+\frac{k a(t)(a(t)-c l)}{c m l^{2}} \chi_{1,1}+\bar{\beta}_{2} \chi_{2,2}^{2}\right.\right. \\
\left.\left.+\frac{k a(t)(a(t)-c l)}{c m l^{2}}\left(K_{2}-K_{1}\right)\right]+l_{2,2}\right\} \mathrm{d} t+\bar{g}_{2,2}\left(\chi_{2,1}, \chi_{2,2}\right) \mathrm{d} w \\
Y_{2}=\chi_{2,1},
\end{array}\right.
\end{aligned}
$$

where $\chi_{i, j}=\log \left(\lambda_{i, j, 1}+x_{i, j} / \lambda_{i, j, 2}-x_{i, j}\right), \quad \bar{g}_{i, j}=-\left(\left(e^{\chi_{i, j}}+1\right)^{2}\right.$ $\left.l e^{\chi_{i, j}}\left(\lambda_{i, j, 2}+\lambda_{i, j, 1}\right)\right) g_{i, j}, \quad l_{i, j}=-\left(\left(e^{\chi_{i, j}}+1\right)^{2}\left(e^{\chi_{i, j}} \dot{\lambda}_{i, j, 2}-\dot{\lambda}_{i, j, 1}\right)\right.$ $\left./ e^{\chi_{i, j}}\left(\lambda_{i, j, 2}+\lambda_{i, j, 1}\right)\left(e^{\chi_{i, j}}+1\right)\right)$, and $h_{i, j}=\left(\left(e^{\chi_{i, j}}+1\right)^{2} / e^{\chi_{i, j}}\left(\lambda_{i, j, 2}\right.\right.$ $\left.\left.+\lambda_{i, j, 1}\right)\right)$ with $i=1$ and 2 and $j=1$ and 2 . The reference signals of the novel closed-loop system are given as

$$
\begin{aligned}
& Y_{1, r}=\log \frac{\lambda_{1,1,1}+y_{1, r}}{\lambda_{1,1,2}-y_{1, r}}, \\
& Y_{2, r}=\log \frac{\lambda_{2,1,1}+y_{2, r}}{\lambda_{2,1,2}-y_{2, r}} .
\end{aligned}
$$

Choose the fuzzy membership functions as follows:

$$
\mu F_{i, j}^{k}=e^{\left[-0.5\left(\chi_{i, j}+6-2 k\right)^{2}\right]}, \quad i=1,2, j=1,2, \ldots, 5 \text {, and } k=1,2, \ldots, 5
$$

Then, the fuzzy basic functions can be constructed as

$$
\varphi_{i, j, k}\left(\underline{\chi}_{i, j}\right)=\frac{\prod_{j=1}^{2} \exp \left[-0.5\left(\underline{\chi}_{i, j}+6-2 k\right)^{2}\right]}{\sum_{k=1}^{5} \prod_{j=1}^{2} \exp \left[-0.5\left(\underline{\chi}_{i, j}+6-2 k\right)^{2}\right]} .
$$

The virtual control functions $\alpha_{i, 1}(i=1$ and 2$)$, the actual control inputs $u_{i}^{*}(i=1$ and 2 ), and the adaptive laws of the parameters $\quad \widehat{W}_{i, 1}(i=1$ and 2$), \quad \widehat{\Theta}_{i, j}(i=1$ and 2 and $j=$ 1 and 2$), \quad \widehat{m}_{i, r}(i=1$ and 2$), \quad \widehat{m}_{i, l}(i=1$ and 2$), \quad \widehat{d}_{i, r, m}(i=1$ and 2$)$, and $\hat{d}_{i, l, m}(i=1$ and 2$)$ are designed as below:

$$
\begin{aligned}
& \alpha_{i, 1}=h_{i, 1}^{-1}\left[-c_{i, 1} z_{i, 1}-\frac{z_{i, 1}^{3} \widehat{\Theta}_{i, 1}}{4 \tau_{i} \varphi_{i, 1}^{T}\left(\bar{X}_{i, 1}\right) \varphi_{i, 1}\left(\bar{X}_{i, 1}\right)}-\frac{z_{i, 1} \widehat{W}_{i}}{4 \tau_{i}}-\frac{z_{i, 1}^{3}+z_{i, 1}}{4 \tau_{i}}\right], \\
& u_{i}^{*}=h_{i, 2}^{-1}\left[-c_{i, 2} z_{i, 2}-\frac{z_{i, 2}^{3} \widehat{\Theta}_{i, 2}}{4 \tau_{i} \varphi_{i, 2}^{T}\left(\bar{X}_{i, 2}\right) \varphi_{i, 2}\left(\bar{X}_{i, 2}\right)}-\frac{z_{i, 2}^{3}}{4 \tau_{i}}-\frac{1}{2} z_{i, 2}^{3} h_{i, 2}^{2}\right],
\end{aligned}
$$

$$
\dot{\widehat{\Theta}}_{i, 1}=\frac{\gamma_{i, 1} z_{i, 1}^{6}}{4 \tau_{i} \varphi_{i, 1}^{T}\left(\bar{X}_{i, 1}\right) \varphi_{i, 1}\left(\bar{X}_{i, 1}\right)}-\sigma_{i, 1} \widehat{\Theta}_{i, 1},
$$$$
\dot{\widehat{\Theta}}_{i, 2}=\frac{\gamma_{i, 2} z_{i, 2}^{6}}{4 \tau_{i} \varphi_{i, 2}^{T}\left(\bar{X}_{i, 2}\right) \varphi_{i, 2}\left(\bar{X}_{i, 2}\right)}-\sigma_{i, 2} \widehat{\Theta}_{i, 2},
$$

$$
\dot{\widehat{W}}_{i}=\frac{\varsigma_{i} z_{i, 1}^{4}}{4 \tau_{i}}-\bar{\sigma}_{i} \widehat{W}_{i}
$$

$\dot{\hat{m}}_{i, r}=\frac{u_{i}^{*}+\widehat{d}_{i, r, m}}{\widehat{m}_{i, r}} \xi_{i, 1} z_{i, 2}^{3} h_{i, 2} \delta_{i}+\bar{\xi}_{i, 1} \widehat{m}_{i, r}$,$$
\dot{\hat{m}}_{i, l}=\frac{u_{i}^{*}+\widehat{d}_{i, l, m}}{\widehat{m}_{i, l}} \xi_{i, 2} z_{i, 2}^{3} h_{i, 2}\left(1-\delta_{i}\right)+\bar{\xi}_{i, 2} \widehat{m}_{i, l},
$$

$\dot{\hat{d}}_{i, r, m}=-\xi_{i, 3} z_{i, 2}^{3} h_{i, 2} \delta_{i}+\bar{\xi}_{i, 3} \widehat{d}_{i, r, m}$,

$\dot{\hat{d}}_{i, l, m}=-\xi_{i, 4} z_{i, 2}^{3} h_{i, 2}\left(1-\delta_{i}\right)+\bar{\xi}_{i, 4} \widehat{d}_{i, l, m}$. 

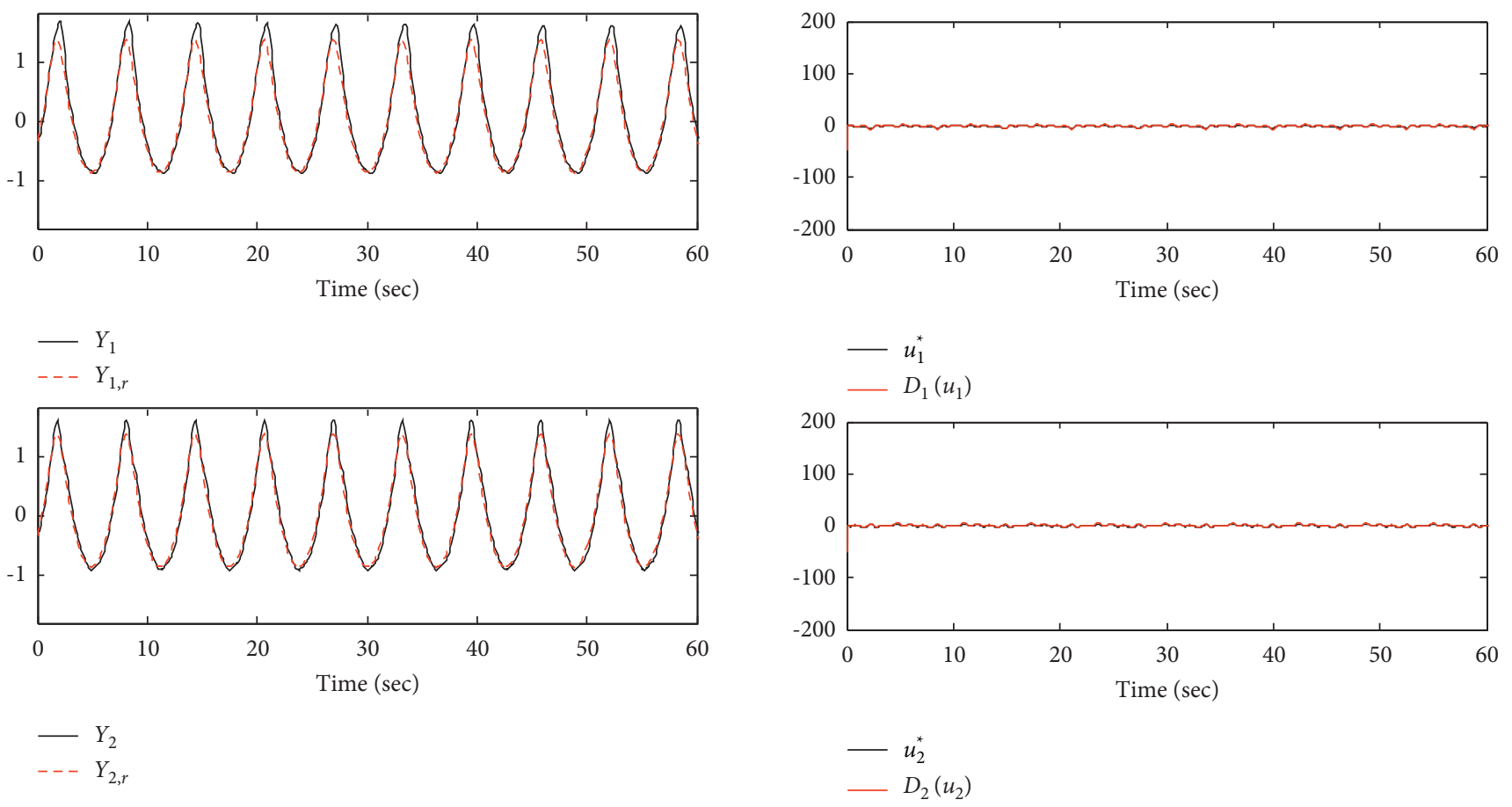

FIGURE 1: Trajectories of outputs $Y_{i}$ and reference signals $Y_{i, r}$.

FIgURE 3: Trajectories of control inputs $u_{i}^{*}$ and dead zones $D_{i}\left(u_{i}\right)$.
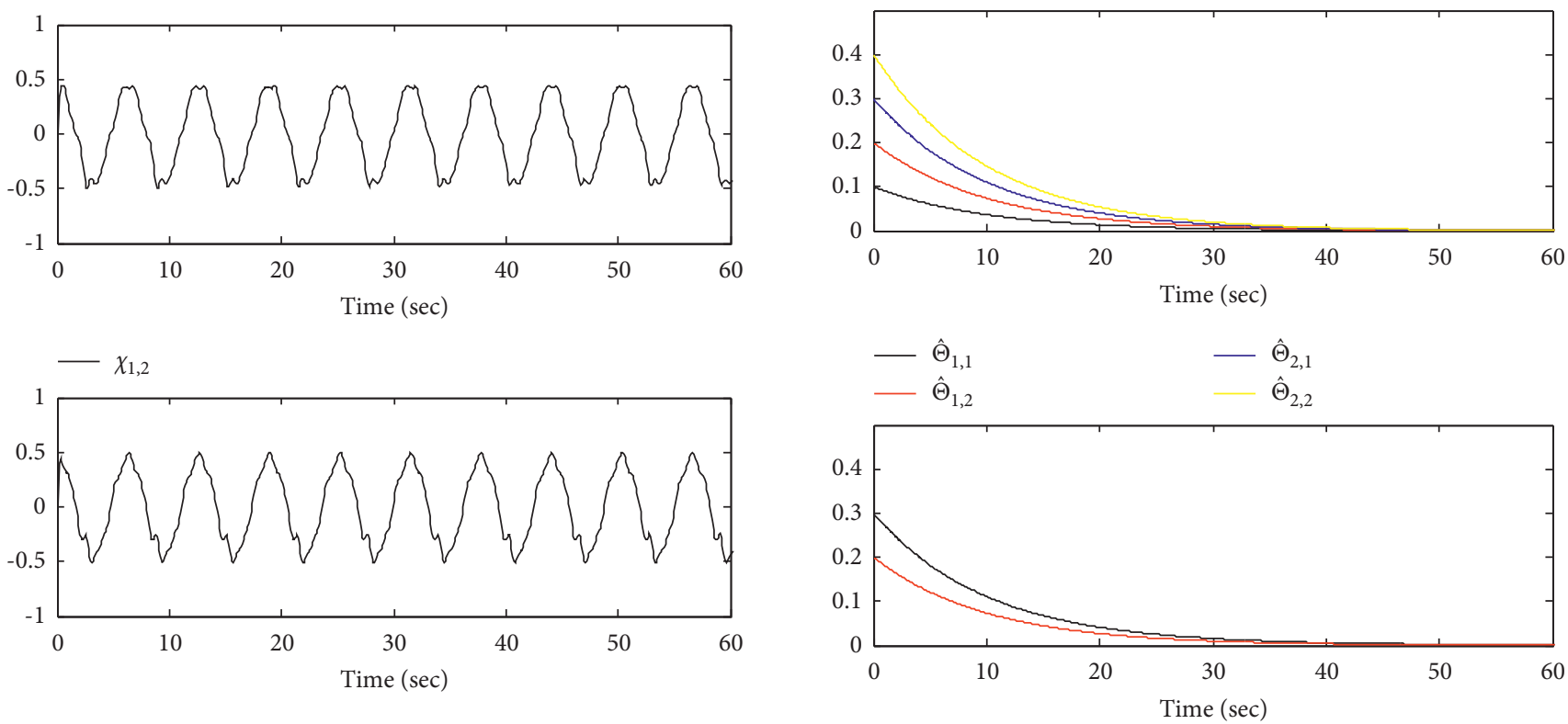

$-\chi_{2,2}$

FIgURE 2: Trajectories of state variables $\chi_{i, 2}$.

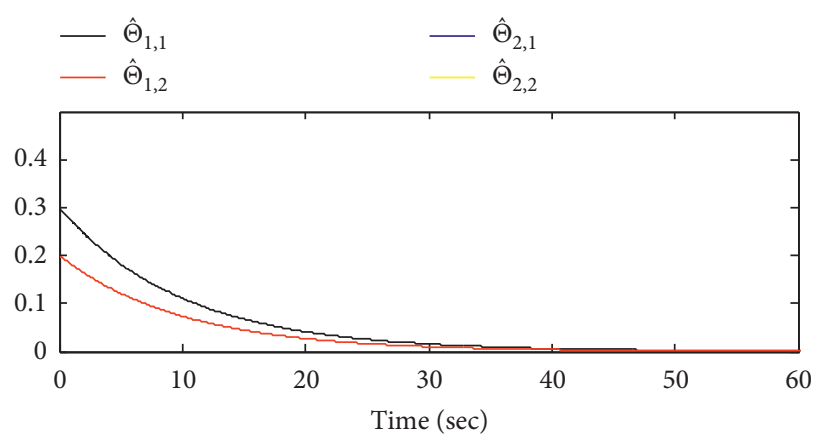

$$
\begin{array}{r}
\hat{W}_{1} \\
-\hat{W}_{2}
\end{array}
$$

FIGURE 4: Trajectories of adaptive parameters $\widehat{\Theta}_{i, j}$ and $\widehat{W}_{i}$. 

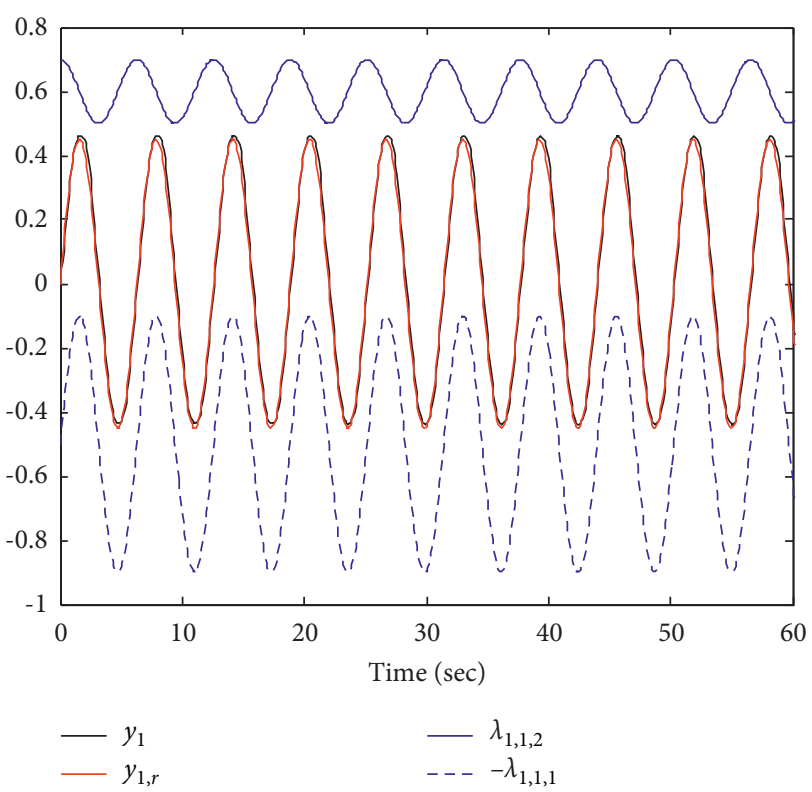

FIGURE 5: Trajectories of output $y_{1}$ and reference signals $y_{1, r}$.

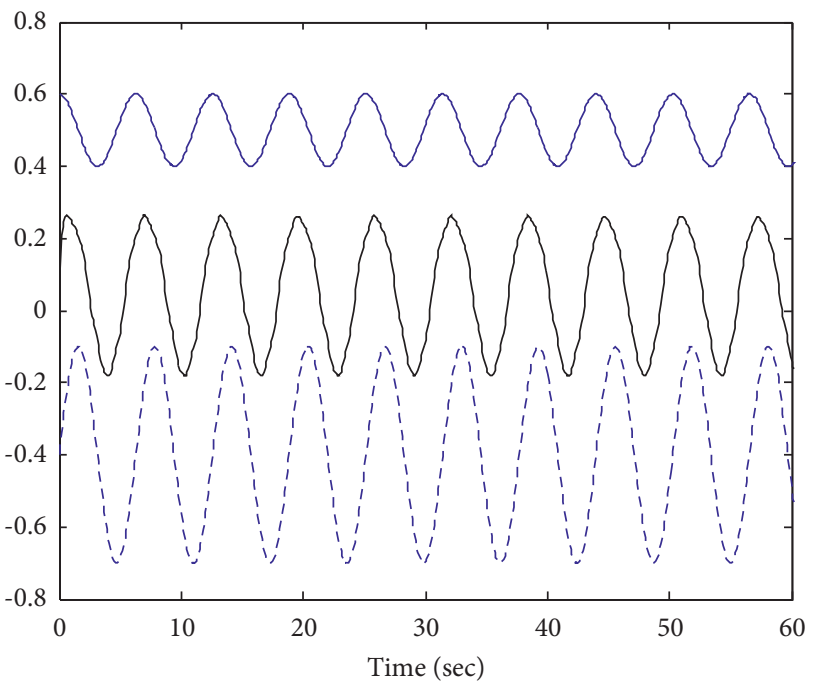

$$
\begin{aligned}
& x_{1,2} \\
& -\lambda_{1,2,2} \\
& -\lambda_{1,2,1}
\end{aligned}
$$

Figure 6: Trajectories of state variable $x_{1,2}$.
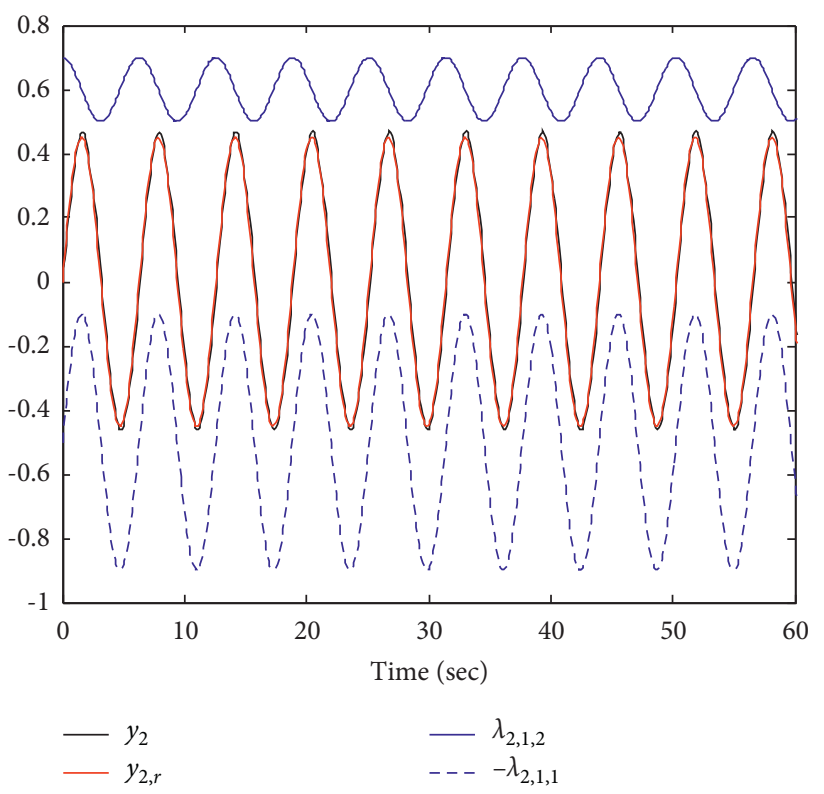

FIgURE 7: Trajectories of output $y_{2}$ and reference signals $y_{2, r}$.

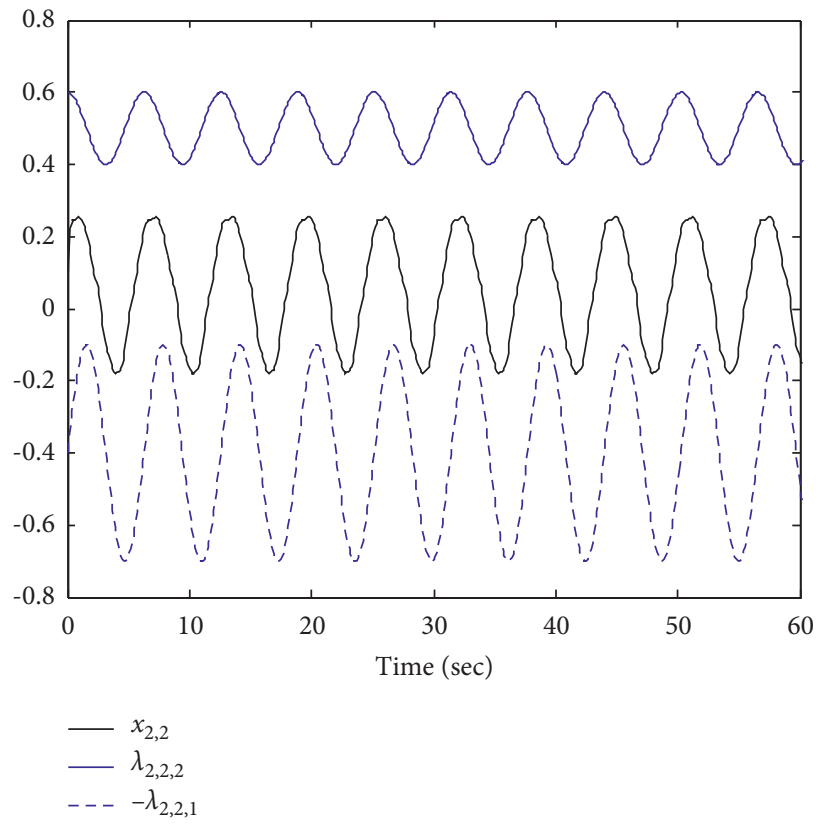

Figure 8: Trajectories of state variable $x_{2,2}$. 


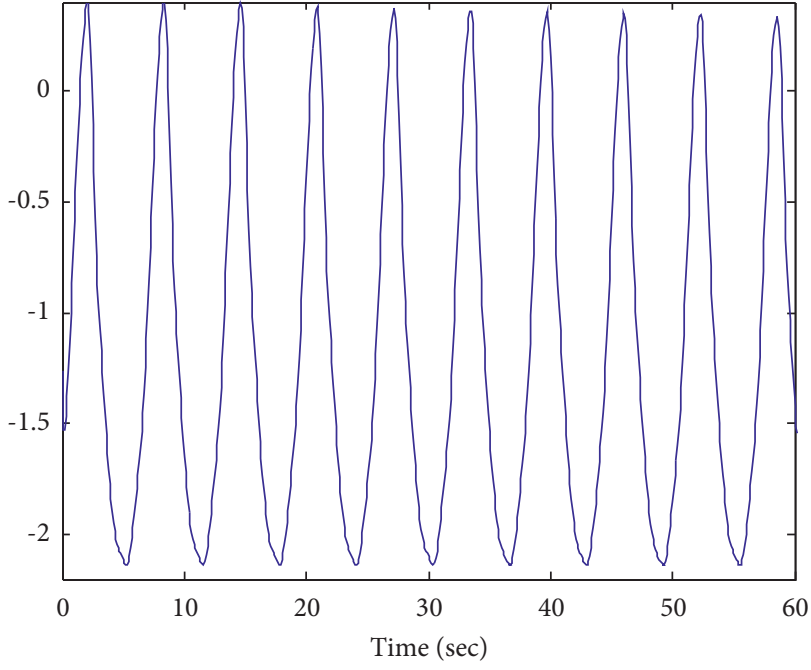

FIGURE 9: Trajectories of tracking errors between $Y_{1}$ and $Y_{1, r}$.

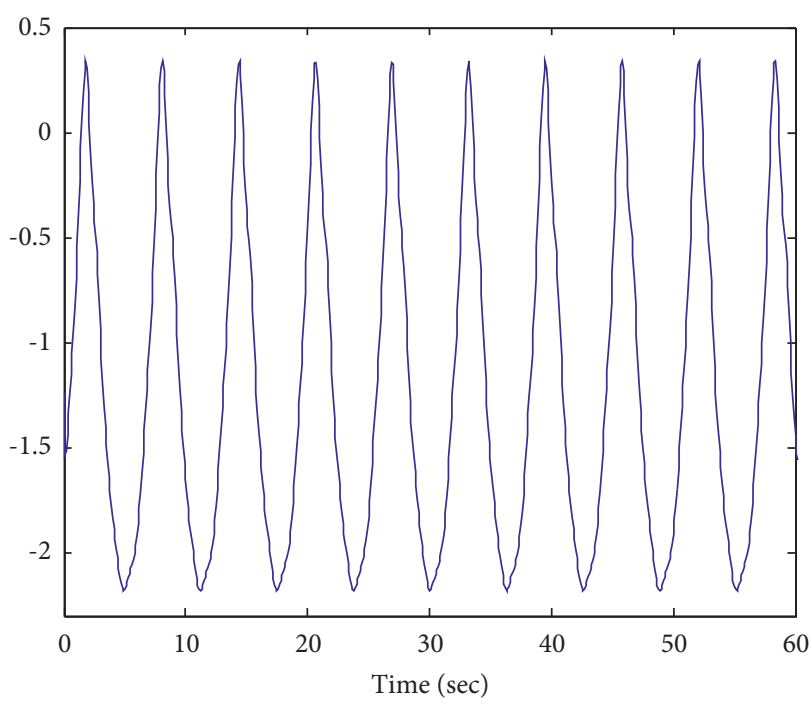

Figure 10: Trajectories of tracking errors between $Y_{2}$ and $Y_{2, r}$.

In the simulation, the initial values are chosen as

$$
\begin{aligned}
{\left[\chi_{1,1}(0), \chi_{1,2}(0), \chi_{2,1}(0), \chi_{2,2}(0)\right]^{T}=} & {[0.01,0.02,0.03,0.01]^{T}, } \\
{\left[\widehat{\Theta}_{1,1}(0), \widehat{\Theta}_{1,2}(0), \widehat{\Theta}_{2,1}(0), \widehat{\Theta}_{2,2}(0)\right]^{T}=} & {[0.1,0.2,0.3,0.4]^{T}, } \\
{\left[\widehat{W}_{1}(0), \widehat{W}_{2}(0)\right]^{T}=} & {[0.3,0.2]^{T}, } \\
& {\left[\widehat{m}_{1, r}(0), \widehat{m}_{1, l}(0), \widehat{d}_{1, r, m}(0), \widehat{d}_{1, l, m}(0), \widehat{m}_{2, r}(0), \widehat{m}_{2, l}(0), \widehat{d}_{2, r, m}(0), \widehat{d}_{2, l, m}(0)\right]^{T} } \\
= & {[1,1,1,1,1,1,1,1]^{T} . }
\end{aligned}
$$

Remark 4. Note that the two inverted pendulums' systems introduced above are free of dead zones and did not consider the stochastic disturbance. However, the stochastic disturbance and dead zones are evitable in practical engineering due to the restrictions of external physical conditions.

The design parameters are selected as

$$
\begin{aligned}
c_{1,1} & =c_{2,1}=30, \\
c_{1,2} & =c_{2,2}=60, \\
\gamma_{1,1} & =\gamma_{1,2}=\gamma_{2,1}=\gamma_{2,2}=0.01, \\
\sigma_{1,1} & =\sigma_{1,2}=\sigma_{2,1}=\sigma_{2,2}=0.01, \\
\varsigma_{1} & =\varsigma_{2}=0.01, \\
\bar{\sigma}_{1} & =\bar{\sigma}_{2}=0.1, \\
\xi_{1,1} & =\xi_{1,2}=\xi_{1,3}=\xi_{1,4}=0.02, \\
\bar{\xi}_{1,1} & =\bar{\xi}_{1,2}=\bar{\xi}_{1,3}=\bar{\xi}_{1,4}=0.001, \\
\xi_{2,1} & =\xi_{2,2}=\xi_{2,3}=\xi_{2,4}=0.02, \\
\bar{\xi}_{2,1} & =\bar{\xi}_{2,2}=\bar{\xi}_{2,3}=\bar{\xi}_{2,4}=0.001 .
\end{aligned}
$$

Insert the control schemes (79)-(87) into systems (74) and (75), and the simulation results are reflected by Figures 1-10. Figure 1 shows the curves of outputs $Y_{i}(i=$
1 and 2) and their reference signals $Y_{i, r}(i=1$ and 2), and the trajectory of outputs $Y_{i}$ track the given signals during the whole period; Figure 2 shows the curves of state variables $\chi_{i, 2}(i=1$ and 2$)$, and it is obvious that $\chi_{i, 2}$ are bounded; both the tracking errors showed in Figures 1 and 2 can be directly reflected by Figures 9 and 10. Figure 3 shows the curves of control inputs $u_{i}(i=1$ and 2$)$ and dead zones $D_{i}\left(u_{i}\right)(i=1$ and 2$)$; Figure 4 shows the curves of adaptive parameters $\widehat{\Theta}_{i, j}(i=1$ and 2 and $j=1$ and 2$) \quad$ and $\widehat{W}_{i}(i=1$ and 2$)$; Figure 5 shows the curves of output $y_{1}$ and its reference signals $y_{1, r}$ in the time-varying constrained closed-loop system; Figure 6 shows the curve of state variable $x_{1,2}$ in the closed-loop system, and it is constrained by time-varying bounds; Figure 7 shows the curves of output $y_{2}$ and its reference signal $y_{2, r}$; Figure 8 shows that the curve of state variable $x_{2,2}$ is constrained by time-varying bounds.

\section{Conclusion}

In this article, the robust adaptive fuzzy control design for stochastic state-constrained nonlinear large-scale systems has been studied. The constrained closed-loop system is transformed into a novel system without any constraint by utilizing the variable transformation method, which can achieve the same control objective. Then, FLSs are used to 
approximate the unknown nonlinear functions, and the dead zone inverse technique is used to compensate the dead zone effect. Combining backstepping design with the decentralized control method, a robust adaptive fuzzy controller is constructed ultimately. In addition, the states of the controlled system remain in their constrained bounds all the time.

\section{Data Availability}

The data used to support the findings of this study are included within the article.

\section{Conflicts of Interest}

The authors declare that they have no conflicts of interest.

\section{Acknowledgments}

This work was supported in part by the National Natural Science Foundation of China under Grants 61973147 and 61803190 and Liaoning Revitalization Talents Program under Grant XLYC1907050.

\section{References}

[1] S. Jain and F. Khorrami, "Decentralized adaptive control of a class of large-scale interconnected nonlinear systems," IEEE Transactions on Automatic Control, vol. 42, no. 2, pp. 136-154, 1997.

[2] L. Shi and S. K. Singh, "Decentralized adaptive controller design for large-scale systems with higher order interconnections," IEEE Transactions on Automatic Control, vol. 37, no. 8, pp. 1106-1118, 1992.

[3] Z. P. Jiang, "Decentralized and adaptive nonlinear tracking of large-scale systems via output feedback," IEEE Transactions on Automatic Control, vol. 45, no. 11, pp. 2122-2128, 2000.

[4] S. Jain and F. Khorrami, "Decentralized adaptive output feedback design for large-scale nonlinear systems," IEEE Transactions on Automatic Control, vol. 42, no. 5, pp. 729735, 1997.

[5] P. Du, H. Liang, S. Zhao, and C. K. Ahn, "Neural-based decentralized adaptive finite-time control for nonlinear largescale systems with time-varying output constraints," IEEE Transactions on Systems, Man, and Cybernetics: Systems, vol. 51, no. 5, pp. 3136-3147, 2021.

[6] Y. Fan, Y. Li, and S. Tong, "Adaptive finite-time fault-tolerant control for interconnected nonlinear systems," International Journal of Robust and Nonlinear Control, vol. 31, no. 5, pp. 1564-1581, 2021.

[7] S. C. Tong, L. L. Zhang, and Y. M. Li, "Observed-based adaptive fuzzy decentralized tracking control for switched uncertain nonlinear large-scale systems with dead zones," IEEE Transactions on Systems, Man, and Cybernetics: Systems, vol. 46, no. 1, pp. 37-47, 2015.

[8] S. Tong, B. Huo, and Y. Li, "Observer-based adaptive decentralized fuzzy fault-tolerant control of nonlinear largescale systems with actuator failures," IEEE Transactions on Fuzzy Systems, vol. 22, no. 1, pp. 1-15, 2014.

[9] T. Liu, Z. P. Jiang, and P. Zhang, "Decentralized eventtriggered control of large-scale nonlinear systems," International Journal of Robust and Nonlinear Control, vol. 30, no. 4, pp. 1451-1466, 2019.
[10] Y. X. Li, S. C. Tong, and G. H. Yang, "Observer-based adaptive fuzzy decentralized event-triggered control of interconnected nonlinear system," IEEE Transactions on Cybernetics, vol. 50, no. 7, pp. 3104-3112, 2019.

[11] D. Yu, H. Xu, C. L. P. Chen, W. Bai, and Z. Wang, "Dynamic coverage control based on K-means," IEEE Transactions on Industrial Electronics, p. 1, 2021.

[12] S. Tong, X. Min, and Y. Li, "Observer-based adaptive fuzzy tracking control for strict-feedback nonlinear systems with unknown control gain functions," IEEE Transactions on Cybernetics, vol. 50, no. 9, pp. 3903-3913, 2020.

[13] Y. L. Wei and H. R. Karimi, "Dynamic sliding mode control for nonlinear parameter-varying systems," International Journal of Robust and Nonlinear Control, 2021.

[14] J. Wu, W. Chen, and J. Li, "Global finite-time adaptive stabilization for nonlinear systems with multiple unknown control directions," Automatica, vol. 69, pp. 298-307, 2016.

[15] S. Sui, C. L. P. Chen, and S. Tong, "Finite-time adaptive fuzzy prescribed performance control for high-order stochastic nonlinear systems," IEEE Transactions on Fuzzy Systems, p. 1, 2021.

[16] H. Deng and M. Krstić, "Stochastic nonlinear stabilization-I: A backstepping design," Systems \& Control Letters, vol. 32, no. 3, pp. 143-150, 1997.

[17] T. Zhang and X. Xia, "Decentralized adaptive fuzzy output feedback control of stochastic nonlinear large-scale systems with dynamic uncertainties," Information Sciences, vol. 315, pp. 17-38, 2015.

[18] S. Tong, S. Sui, and Y. Li, "Adaptive fuzzy decentralized tracking fault-tolerant control for stochastic nonlinear largescale systems with unmodeled dynamics," Information Sciences, vol. 289, pp. 225-240, 2014.

[19] S. Tong, S. Sui, and Y. Li, "Adaptive fuzzy decentralized output stabilization for stochastic nonlinear large-scale systems with unknown control directions," IEEE Transactions on Fuzzy Systems, vol. 22, no. 5, pp. 1365-1372, 2014.

[20] S. C. Tong, Y. M. Li, and T. Wang, "Adaptive fuzzy decentralized output feedback control for stochastic nonlinear large-scale systems using DSC technique," International Journal of Robust and Nonlinear Control, vol. 30, no. 4, pp. 1451-1466, 2019.

[21] K. P. Tee, S. S. Ge, and E. H. Tay, "Barrier Lyapunov functions for the control of output-constrained nonlinear systems," Automatica, vol. 45, no. 4, pp. 918-927, 2009.

[22] K. P. Tee, B. Ren, and S. S. Ge, "Control of nonlinear systems with time-varying output constraints," Automatica, vol. 47, no. 11, pp. 2511-2516, 2011.

[23] Y.-J. Liu, S. Lu, S. Tong, X. Chen, C. L. P. Chen, and D.-J. Li, "Adaptive control-based Barrier Lyapunov functions for a class of stochastic nonlinear systems with full state constraints," Automatica, vol. 87, pp. 83-93, 2018.

[24] Y.-J. Liu and S. Tong, "Barrier Lyapunov functions for Nussbaum gain adaptive control of full state constrained nonlinear systems," Automatica, vol. 76, pp. 143-152, 2017.

[25] Y.-J. Liu and S. Tong, "Barrier Lyapunov Functions-based adaptive control for a class of nonlinear pure-feedback systems with full state constraints," Automatica, vol. 64, pp. 70-75, 2016.

[26] D. Yu, C. L. P. Chen, and H. Xu, "Fuzzy swarm control based on sliding-mode strategy with self-organized omnidirectional mobile robots system," IEEE Transactions on Systems, Man, and Cybernetics: Systems, pp. 1-13, 2021.

[27] J. Wu, Z.-G. Wu, J. Li, G. Wang, H. Zhao, and W. Chen, "Practical adaptive fuzzy control of nonlinear pure-feedback 
systems with quantized nonlinearity input," IEEE Transactions on Systems, Man, and Cybernetics: Systems, vol. 49, no. 3, pp. 638-648, 2019.

[28] S. Sui, C. L. P. Chen, and S. C. Tong, "Neural-network-based adaptive DSC design for switched fractional-order nonlinear systems," IEEE Transactions on Neural Networks and Learning Systems, vol. 32, no. 10, pp. 4703-4712, 2021.

[29] G. Lin, H. Li, H. Ma, D. Yao, and R. Lu, "Human-in-the-loop consensus control for nonlinear multi-agent systems with actuator faults," IEEE/CAA Journal of Automatica Sinica, pp. 1-12, 2020.

[30] Y. J. Liu, J. Li, S. C. Tong, and C. L. P. Chen, "Neural network control-based adaptive learning design for nonlinear systems with full-state constraints," IEEE Transactions on Neural Networks and Learning Systems, vol. 27, no. 7, pp. 1562-1571, 2015.

[31] T. Zhang, M. Xia, and Y. Yi, “Adaptive neural dynamic surface control of strict-feedback nonlinear systems with full state constraints and unmodeled dynamics," Automatica, vol. 81, pp. 232-239, 2017.

[32] K. Zhao, Y. D. Song, T. D. Ma, and L. He, “"Prescribed performance control of uncertain euler-Lagrange systems subject to full-state constraints", IEEE Transactions on Neural Networks and Learning Systems, vol. 29, no. 8, pp. 3478-3489, 2017.

[33] L. Fang, S. Ding, J. H. Park, and L. Ma, “Adaptive fuzzy control for nontriangular stochastic high-order nonlinear systems subject to asymmetric output constraints," IEEE Transactions on Cybernetics, pp. 1-12, 2020.

[34] K. Zhao, Y. Song, C. L. P. Chen, and L. Chen, "Control of nonlinear systems under dynamic constraints: a unified barrier function-based approach," Automatica, vol. 119, Article ID 109102, 2020.

[35] K. Zhao, Y. Song, and Z. Zhang, "Tracking control of MIMO nonlinear systems under full state constraints: a Single-parameter adaptation approach free from feasibility conditions," Automatica, vol. 107, pp. 52-60, 2019.

[36] Y. Cao, Y. Song, and C. Wen, "Practical tracking control of perturbed uncertain nonaffine systems with full state constraints," Automatica, vol. 110, Article ID 108608, 2019.

[37] T. Jia, Y. Pan, H. Liang, and H.-K. Lam, "Event-based adaptive fixed-time fuzzy control for active vehicle suspension systems with time-varying displacement constraint," IEEE Transactions on Fuzzy Systems, p. 1, 2021.

[38] K. Zhao and Y. Song, "Neuroadaptive robotic control under time-varying asymmetric motion constraints: a FeasibilityCondition-Free Approach," IEEE Transactions on Cybernetics, vol. 50, no. 1, pp. 15-24, 2020.

[39] H. Li, S. Zhao, W. He, and R. Lu, "Adaptive finite-time tracking control of full state constrained nonlinear systems with dead-zone,” Automatica, vol. 100, pp. 99-107, 2019.

[40] Q. Zhou, S. Zhao, H. Li, R. Lu, and C. Wu, "Adaptive neural network tracking control for robotic manipulators with dead zone," IEEE Transactions on Neural Networks and Learning Systems, vol. 30, no. 12, pp. 3611-3620, 2019.

[41] Q. Chen, L. Yu, and Y. Nan, "Finite-time tracking control for motor servo systems with unknown dead-zones," Journal of Systems Science and Complexity, vol. 26, no. 6, pp. 940-956, 2013.

[42] T. P. Zhang and S. S. Ge, "Adaptive dynamic surface control of nonlinear systems with unknown dead zone in pure feedback form," Automatica, vol. 44, no. 7, pp. 1895-1903, 2007.

[43] R. Shahnazi, "Cooperative neuro adaptive control of leader following uncertain multi-agent systems with unknown hysteresis and dead-zone," Journal of Systems Science and Complexity, vol. 33, no. 2, pp. 312-332, 2020.

[44] S. Tong, Y. Li, and S. Sui, "Adaptive fuzzy output feedback control for switched nonstrict-feedback nonlinear systems with input nonlinearities," IEEE Transactions on Fuzzy Systems, vol. 24, no. 6, pp. 1426-1440, 2016.

[45] Y. He, J. Wang, and R. Hao, "Adaptive robust dead-zone compensation control of electro-hydraulic servo systems with load disturbance rejection," Journal of Systems Science and Complexity, vol. 28, no. 2, pp. 341-359, 2015.

[46] B. Bing Chen, X. P. Liu, S. S. Ge, and C. Lin, "Adaptive fuzzy control of a class of nonlinear systems by fuzzy approximation approach," IEEE Transactions on Fuzzy Systems, vol. 20, no. 6, pp. 1012-1021, 2012.

[47] H. Wang, X. Liu, K. Liu, and H. R. Karimi, "Approximationbased adaptive fuzzy tracking control for a class of nonstrictfeedback stochastic nonlinear time-delay systems," IEEE Transactions on Fuzzy Systems, vol. 23, no. 5, pp. 1746-1760, 2015.

[48] B. Chen, H. Zhang, and C. Lin, "Observer-based adaptive neural network control for nonlinear systems in nonstrictfeedback form," IEEE Transactions on Neural Networks and Learning Systems, vol. 27, no. 1, pp. 89-98, 2016.

[49] S. Tong, Y. Li, and S. Sui, "Adaptive fuzzy tracking control design for SISO uncertain nonstrict feedback nonlinear systems," IEEE Transactions on Fuzzy Systems, vol. 24, no. 6, pp. 1441-1454, 2016.

[50] Y. Li and S. Tong, "Adaptive neural networks decentralized FTC design for nonstrict-feedback nonlinear interconnected large-scale systems against actuator faults," IEEE Transactions on Neural Networks and Learning Systems, vol. 28, no. 11, pp. 2541-2554, 2017.

[51] Z. Cao, Y. Niu, and H. Reza Karimi, "Dynamic output feedback sliding mode control for Markovian jump systems under stochastic communication protocol and its application," International Journal of Robust and Nonlinear Control, vol. 30, no. 17, pp. 7307-7325, 2020.

[52] W. Sun, J. Xia, and Y. Wu, "Adaptive tracking control for mobile manipulators with stochastic disturbances," Journal of Systems Science and Complexity, vol. 32, no. 5, pp. 1393-1403, 2019.

[53] J. Wu, X. Chen, Q. Zhao, J. Li, and Z.-G. Wu, “Adaptive neural dynamic surface control with prespecified tracking accuracy of uncertain stochastic nonstrict-feedback systems," IEEE Transactions on Cybernetics, pp. 1-14, 2020.

[54] H. Liang, G. Liu, T. Huang, H.-K. Lam, and B. Wang, "Cooperative fault-tolerant control for networks of stochastic nonlinear systems with nondifferential saturation nonlinearity," IEEE Transactions on Systems, Man, and Cybernetics: Systems, pp. 1-11, 2020.

[55] L. Qiu, L. Guo, and J. Liu, "Mean square average generalized consensus of multi-agent systems under time-delays and stochastic disturbances," Journal of Systems Science and Complexity, vol. 32, no. 2, pp. 588-599, 2019.

[56] Y. Liu, H. Ma, and H. Ma, "Adaptive fuzzy fault-tolerant control for uncertain nonlinear switched stochastic systems with time-varying output constraints," IEEE Transactions on Fuzzy Systems, vol. 26, no. 5, pp. 2487-2498, 2018.

[57] B. Niu, Y. Liu, G. Zong, Z. Han, and J. Fu, "Command filterbased adaptive neural tracking controller design for uncertain switched nonlinear output-constrained systems," IEEE Transactions on Cybernetics, vol. 47, no. 10, pp. 3160-3171, 2017. 